\title{
BMJ Open Gonadotropin-releasing hormone antagonists versus standard androgen suppression therapy for advanced prostate cancer A systematic review with meta-analysis
}

\author{
Frank Kunath, ${ }^{1,2}$ Hendrik Borgmann, ${ }^{2,3}$ Anette Blümle, ${ }^{4}$ Bastian Keck, ${ }^{1}$ \\ Bernd Wullich, ${ }^{1,2}$ Christine Schmucker, ${ }^{4}$ Danijel Sikic, ${ }^{1}$ Catharina Roelle, ${ }^{1}$ \\ Stefanie Schmidt, ${ }^{2}$ Amr Wahba, ${ }^{5}$ Joerg J Meerpohl ${ }^{4}$
}

To cite: Kunath $F$,

Borgmann $\mathrm{H}$, Blümle $\mathrm{A}$, et al. Gonadotropin-releasing hormone antagonists versus standard androgen suppression therapy for advanced prostate cancer $\mathrm{A}$ systematic review with metaanalysis. BMJ Open 2015;5: e008217. doi:10.1136/ bmjopen-2015-008217

- Prepublication history for this paper is available online. To view these files please visit the journal online (http://dx.doi.org/10.1136/ bmjopen-2015-008217).

Received 10 May 2015 Revised 12 September 2015 Accepted 9 October 2015

CrossMark

For numbered affiliations see end of article.

Correspondence to Dr Frank Kunath; frank. kunath@uk-erlangen.de

\section{ABSTRACT}

Objectives: To evaluate efficacy and safety of gonadotropin-releasing hormone $(\mathrm{GnRH})$ antagonists compared to standard androgen suppression therapy for advanced prostate cancer.

Setting: The international review team included methodologists of the German Cochrane Centre and clinical experts.

Participants: We searched CENTRAL, MEDLINE, Web of Science, EMBASE, trial registries and conference books for randomised controlled trials (RCT) for effectiveness data analysis, and randomised or nonrandomised controlled studies (non-RCT) for safety data analysis (March 2015). Two authors independently screened identified articles, extracted data, evaluated risk of bias and rated quality of evidence according to GRADE.

Results: 13 studies (10 RCTs, 3 non-RCTs) were included. No study reported cancer-specific survival or clinical progression. There were no differences in overall mortality (RR $1.35,95 \% \mathrm{Cl} 0.63$ to 2.93 ), treatment failure (RR $0.91,95 \% \mathrm{Cl} 0.70$ to 1.17 ) or prostate-specific antigen progression (RR 0.83, 95\% $\mathrm{Cl} 0.64$ to 1.06). While there was no difference in quality of life related to urinary symptoms, improved quality of life regarding prostate symptoms, measured with the International Prostate Symptom Score (IPSS), with the use of GnRH antagonists compared with the use of standard androgen suppression therapy (mean score difference $-0.40,95 \% \mathrm{Cl}-0.94$ to 0.14 , and $-1.84,95 \% \mathrm{Cl}-3.00$ to -0.69 , respectively) was found. Quality of evidence for all assessed outcomes was rated low according to GRADE. The risk for injection-site events was increased, but cardiovascular events may occur less often by using $\mathrm{GnRH}$ antagonist. Available evidence is hampered by risk of bias, selective reporting and limited follow-up.

Conclusions: There is currently insufficient evidence to make firm conclusive statements on the efficacy of $\mathrm{GnRH}$ antagonist compared to standard androgen suppression therapy for advanced prostate cancer.

\section{Strengths and limitations of this study}

- We searched CENTRAL, MEDLINE, Web of Science, EMBASE, trial registries and conference books. Two authors independently screened identified articles, extracted data, evaluated risk of bias and rated quality of evidence according to GRADE.

- There were no statistically significant differences in overall mortality, treatment failure, or prostatespecific antigen progression and no study reported cancer-specific survival or clinical progression.

- Quality of evidence for all assessed outcomes was rated low according to GRADE. Available evidence is hampered by risk of bias, selective reporting and limited follow-up.

- The question that was addressed by this systematic review was in some points different from the available evidence. There is currently insufficient evidence to make firm conclusive statements on the efficacy of GnRH antagonist compared to standard androgen suppression therapy for advanced prostate cancer and there is a need for further high quality research on GnRH antagonists with long-term follow-up.

There is need for further high-quality research on GnRH antagonists with long-term follow-up.

Trial registration number: CRD42012002751.

\section{INTRODUCTION}

Gonadotropin-releasing hormone (GnRH) antagonists, such as abarelix or degarelix, are new agents for androgen suppression therapy in advanced prostate cancer. They act by competitively binding to receptors in the pituitary gland, leading to reduced amounts 
of luteinising hormone and follicle-stimulating hormone. GnRH antagonists are, thereby, able to decrease the level of testosterone immediately to castration levels without flare. ${ }^{1-3}$ Testosterone is important for the growth of prostate cells and its suppression slows down the disease progression and leads to a decrease in prostate-specific antigen (PSA).

Data from published randomised controlled trials support the use of degarelix as an alternative to standard androgen suppression therapies. ${ }^{45}$ Abarelix appears to be equally effective. ${ }^{2}{ }^{6}$ Androgen suppression therapy with degarelix may also be more cost-effective in patients with locally advanced prostate cancer $^{7-9}$ and may increase PSA progression-free and overall survival. ${ }^{5} 10$ Additionally, degarelix might also have beneficial effects on lower urinary tract symptoms. ${ }^{11}$ Furthermore, GnRH antagonists might provide an alternative to castration in symptomatic patients with advanced prostate cancer because there is no risk for testosterone flare associated with GnRH agonists that might aggravate clinical symptoms. ${ }^{12}$ Despite these positive findings, the current European guideline indicate that there is no definitive evidence that GnRH antagonists have advantages over GnRH agonists. ${ }^{13}$

An analysis of pooled individual patient data of five randomised clinical trials found clinical benefits with degarelix compared with GnRH agonists. ${ }^{10}$ However, no systematic review based on a comprehensive literature search using predefined methodology have yet evaluated the efficacy and tolerability of GnRH antagonists in comparison with standard androgen suppression therapy for advanced prostate cancer. Therefore, the objectives of this systematic review are to determine the efficacy and safety of GnRH antagonists compared with standard androgen suppression therapy for advanced prostate cancer treatment.

\section{METHODS}

For details on our predefined methodology and outcomes see the prospective registry entry in the 'International Prospective Register of Systematic Reviews' (http://www.crd.york.ac.uk/PROSPERO;CRD 42012002751).

We included studies that compared GnRH antagonists (abarelix and degarelix) with standard androgen suppression therapy in patients with advanced prostate cancer. Included studies had to be randomised controlled trials (that were used for efficacy and safety analysis) or prospective non-randomised controlled studies (that were used for adverse events and quality of life analysis). If randomised controlled trials were identified with cross-over design, we only included the data just before cross-over started. We did not exclude studies because of publication status or language of publication, nor did we make restrictions based on age or ethnicity of patients.

We included all patients with advanced prostate cancer. Advanced disease was defined as either locally advanced (T3-4, N0, M0), local to regionally advanced (T1-4, N1, M0), disseminated disease (T1-4, N0-1, M1) or PSA relapse after local therapy.

Included studies had to compare GnRH antagonists (abarelix or degarelix) with standard androgen suppression. The standard androgen suppression therapy included monotherapy with surgical or medical castration, antiandrogen monotherapy or maximal androgen blockade (combination of either surgical or medical castration with antiandrogens).

Our prospectively defined primary outcomes were overall survival and adverse events. We defined cancerspecific survival, clinical or PSA progression, treatment failure and quality of life as secondary outcomes. No study was excluded solely because the outcome of interest was not reported.

Unit of analysis was the study rather than publications, and we named the studies according to their study identification numbers assigned by the sponsors. We used the sponsors identification numbers for differentiation because several authors were involved in more than one study, publications were identified reporting information on several studies (pooled analyses of individual patient data of five randomised controlled trials: CS21, CS28, CS30, CS31, CS35), and as there were several publications available for some studies (eg, different follow-up time or reporting different outcomes).

We searched the Cochrane Library (CENTRAL, Issue 3, 2015), MEDLINE (via Ovid; 1946 to March 2015), Web of Science (Thomson Reuters Web of Knowledge; 1970 to March 2015), and EMBASE (via DIMDI; 1947 to March 2015) databases. For details on the search strategy, see table 1 .

Additionally, we searched three trial registries: Current Controlled Trials (ISRCTN; http://www.controlled-trials. com/; last search in March 2015), ClinicalTrials.gov (http://www.clinicaltrials.gov/; last search in March 2015), and the WHO International Clinical Trials Registry Platform Search Portal (WHO ICTRP Search Portal; http://www.who.int/ictrp/en/; last search in March 2015). We used the following keywords for this search: 'abarelix', 'degarelix', 'plenaxis', 'firmagon'.

We also searched the electronically available abstract books from three major conferences: American Society of Clinical Oncology (ASCO; jco.ascopubs.org; 2004 to March 2015), European Association of Urology (EAU; http://www.uroweb.org; 2004 to March 2015), and American Urological Association (AUA; http://www. jurology.com/; 2008 to March 2015). We used the following keywords for this search: 'abarelix', 'degarelix', 'plenaxis', 'firmagon'.

Furthermore, reference lists of retrieved articles were also searched manually. We also used the safety data analyses from the websites of the Food and Drug Administration (FDA), and the European Medicines Agency (EMEA) to obtain additional information on studies that included patients treated with $\mathrm{GnRH}$ antagonists. 
Table 1 Search strategy

\begin{tabular}{|c|c|c|}
\hline $\begin{array}{l}\text { CENTRAL } \\
\text { (The Cochrane } \\
\text { Library) }\end{array}$ & $\begin{array}{l}1 \\
2\end{array}$ & $\begin{array}{l}\text { MeSH descriptor: (prostatic neoplasms) explode all trees } \\
\text { (prostat* near (cancer* or tumo* or neoplas* or carcinom* or malign*)) }\end{array}$ \\
\hline \multirow[t]{8}{*}{ 03/2015 } & 3 & $(\# 1$ or \#2) \\
\hline & 4 & (LHRH antagonist* or LH RH antagonist* or GNRH antagonist* or GN RH antagonist*) \\
\hline & 5 & $\left(\right.$ FE200486* or FE $\left.200486^{\star}\right)$ \\
\hline & 6 & (firmagon* or degarelix*) \\
\hline & 7 & (PPI149* or PPI $\left.149^{\star}\right)$ \\
\hline & 8 & $\left(\right.$ abarelix $^{\star}$ or plenaxis $\left.{ }^{*}\right)$ \\
\hline & 9 & (\#4 or \#5 or \#6 or \#7 or \#8) \\
\hline & 10 & (\#3 and \#9) \\
\hline MEDLINE (Ovid) & 1 & Prostatic neoplasms/ \\
\hline \multirow[t]{9}{*}{$1946-03 / 2015$} & 2 & (prostat ${ }^{\star}$ adj3 (cancer* or tumo* or neoplas* or carcinom* or malign $\left.{ }^{\star}\right)$ ).tw. \\
\hline & 3 & 1 or 2 \\
\hline & 4 & 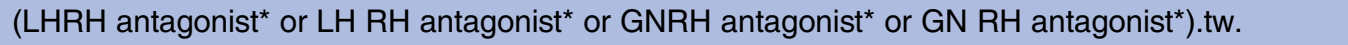 \\
\hline & 5 & $\left(\mathrm{FE} 200486^{\star}\right.$ or FE $\left.200486^{\star}\right) \cdot \mathrm{mp}$ \\
\hline & 6 & (firmagon* or degarelix $\left.{ }^{\star}\right) \cdot m p$ \\
\hline & 7 & $\left(\mathrm{PPI} 149^{\star}\right.$ or PPI $\left.149^{\star}\right) \cdot \mathrm{mp}$ \\
\hline & 8 & (abarelix* or plenaxis*).mp. \\
\hline & 9 & 4 or 5 or 6 or 7 or 8 \\
\hline & 10 & 3 and 9 \\
\hline EMBASE (DIMDI) & 1 & EM74 \\
\hline \multirow[t]{10}{*}{$1947-03 / 2015$} & 2 & $\begin{array}{l}\text { CT=("PROSTATE TUMOR"; "PROSTATE CANCER"; "PROSTATE ADENOCARCINOMA"; } \\
\text { "PROSTATE CARCINOMA)" }\end{array}$ \\
\hline & 3 & (prostat ${ }^{\star}$ and (cancer ${ }^{\star}$ or tumo* or neoplas* or carcinom* or malign*))/same sent \\
\hline & 4 & 2 OR 3 \\
\hline & 5 & (LHRH antagonist* or LH RH antagonist* or GNRH antagonist* or GN RH antagonist $\left.{ }^{\star}\right) /$ same sent \\
\hline & 6 & $\left(\right.$ FE200486* or FE $\left.200486^{\star}\right) /$ same sent \\
\hline & 7 & (firmagon* or degarelix*)/same sent \\
\hline & 8 & $\left(\mathrm{PPI} 149^{\star}\right.$ or PPI $\left.149^{\star}\right) /$ same sent \\
\hline & 9 & $\left(\right.$ abarelix $^{*}$ or plenaxis*)/same sent \\
\hline & 10 & 5 OR 6 OR 7 OR 8 OR 9 \\
\hline & 11 & 4 AND 10 \\
\hline Web of Science & 1 & TS $=\left(\right.$ prostat $^{\star}$ same $\left(\right.$ cancer $^{\star}$ or tumo* or neoplas ${ }^{*}$ or carcinom ${ }^{\star}$ or malign $\left.\left.{ }^{\star}\right)\right)$ \\
\hline \multirow[t]{10}{*}{$1970-03 / 2015$} & 2 & $\mathrm{TS}=\left(\left(\right.\right.$ LHRH same antagonist $\left.^{\star}\right)$ or $\left(\right.$ LH same RH same antagonist $\left.\left.{ }^{\star}\right)\right)$ \\
\hline & 3 & TS $=\left(\left(\right.\right.$ gnrh same antagonist $\left.{ }^{\star}\right)$ OR (gn same rh same antagonist $\left.\left.{ }^{\star}\right)\right)$ \\
\hline & 4 & TS $=($ FE200486*) \\
\hline & 5 & $\mathrm{TS}=\left(\mathrm{FE}\right.$ same $\left.200486^{\star}\right)$ \\
\hline & 6 & TS $=\left(\right.$ abarelix ${ }^{\star}$ OR plenaxis $\left.{ }^{\star}\right)$ \\
\hline & 7 & TS=(firmagon* OR degarelix*) \\
\hline & 8 & $\mathrm{TS}=\left(\mathrm{PPI} 149^{*}\right)$ \\
\hline & 9 & $\mathrm{TS}=\left(\mathrm{PPI}\right.$ same $\left.149^{\star}\right)$ \\
\hline & 10 & \#9 OR \#8 OR \#7 OR \#6 OR \#5 OR \#4 OR \#3 OR \#2 \\
\hline & 11 & \#10 AND \#1 \\
\hline
\end{tabular}

The search of all databases was initially conducted in March 2014 and was updated in March 2015. The search update included only studies that were published since our initial search (studies published from March 2014 to March 2015). No language restrictions were applied.

Two authors independently screened retrieved references for inclusion (FK, HB), and two authors (FK, AB) independently extracted data using standardised data extraction forms and assessed each study's risk of bias. We resolved any disagreements through double-checking the respective articles, or through discussion with a third review author $(\mathrm{JM})$. One review author performed the search update (FK). Randomised studies' risk of bias was assessed following the recommendations of the Cochrane Handbook by Higgins et $a l^{14}$ We used the checklist recommended by Reeves $e t a l^{15}$ for data collection and study assessment for non-randomised studies.

We used the Cochrane RevMan V.5.2 for statistical data analyses (http://tech.cochrane.org/revman/), and the GRADE working group's software GRADEpro to develop the GRADE evidence table (http://www. gradeworkinggroup.org/). ${ }^{16}{ }^{17}$ We identified no studies evaluating time-to-event outcomes. Therefore, no HRs were extracted.

We extracted outcomes data relevant to this review, as needed, for calculation of summary statistics and 
measures of variance. For dichotomous outcomes, we attempted to obtain numbers of events and totals to calculate pooled risk ratios (RRs) with their 95\% CIs using Mantel-Haenszel method. Continuous outcomes were analysed using the inverse variance method and were expressed as mean differences (MD) with 95\% CI. We defined $\mathrm{p}<0.05$ as statistically significant. We assessed statistical heterogeneity among studies $\left(\chi^{2}, \mathrm{I}^{2}\right)$ and employed a fixed effects model for $\mathrm{I}^{2} \leq 50 \%$ and additionally, a random effects model for $\mathrm{I}^{2}>50 \%$ for use in a sensitivity analysis.

We performed subgroup analyses for the different doses of androgen suppression therapy and for the different GnRH antagonists (abarelix and degarelix). Initially, we also planned to perform subgroup analyses for non-metastatic versus metastatic disease. However, results were not reported for these subgroups in the included studies.

\section{RESULTS}

\section{Study characteristics}

We identified 15 studies but only 13 (10 randomised and 3 non-randomised controlled trials) were included in this review. Two of the three non-randomised studies were cross-over studies (Zuckerman 2013, Garnick 2011). See figures 1 and 2 for details regarding the literature search.

Abarelix depot $100 \mathrm{mg}$ intramuscularly administered on day 0 , day 15 and every 4 weeks thereafter was evaluated in six studies:

- $149-97-04^{1} 1920$
- $149-98-02^{6} 21-23$
- $149-98-03^{2} 22-26$
- $149-99-03^{23} 27$
- ABACS1 ${ }^{23} 28-30$
- Garnick $2011^{31}$

Seven studies evaluated degarelix $240 \mathrm{mg}$ subcutaneously administered as a starting dose, and 80 or $160 \mathrm{mg}$ subcutaneous maintenance doses every 4 weeks thereafter:

- CS21 ${ }^{10} 32-63$

- CS28 $8^{10} 32-35$ 60-62 64-66

- CS30 ${ }^{10} 32-35$ 60-62 65-68

- CS3110 32-35 60-62 65666970

- CS35 ${ }^{10}$ 32-35 59-62

- CS37 $7^{32-35}$ 60-62

- Zuckerman $2013^{71} 72$

The two excluded studies were retrieved from the FDA website (149-01-03 and 149-01-05). We identified no publications regarding these studies and were, therefore, not able to include the studies in our analyses because we found no further methodological information or study results. Study 149-01-03 was an open-label trial that compared neoadjuvant hormonal therapy with abarelix depot $100 \mathrm{mg}$ intramuscularly with leuprolide depot $7.5 \mathrm{mg}$ intramuscularly in patients with prostate cancer who planned to undergo brachytherapy or external-

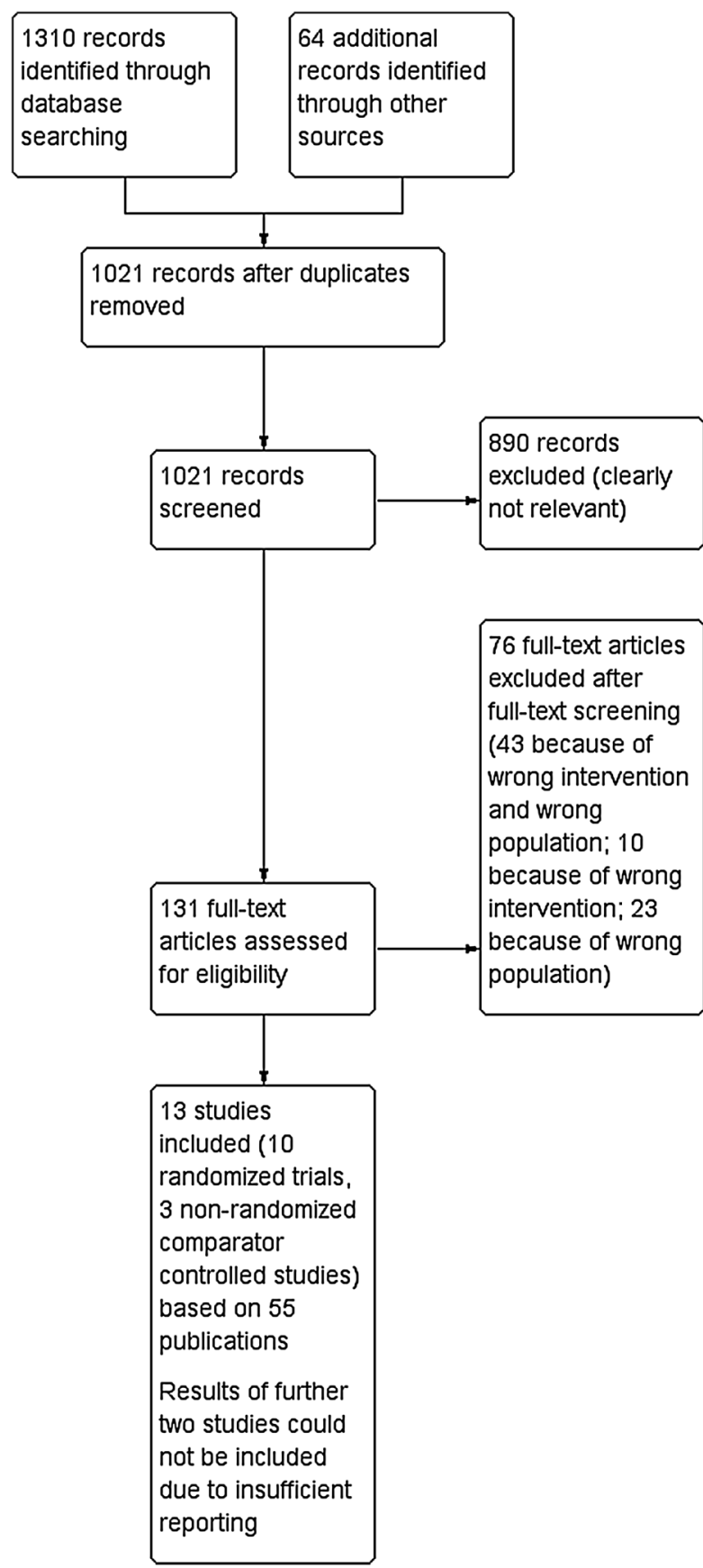

Figure 1 Flow chart of initially search in March 2014; adapted to the flow chart recommended by Liberati et al. ${ }^{18}$

beam radiation therapy. ${ }^{23}$ Study 149-01-05 was an openlabel cross-over study to evaluate the feasibility of switching to treatment with a GnRH agonist following 12 weeks of treatment with abarelix in patients with prostate cancer. $^{23}$

The 13 included studies resulted in 55 citations (16 full journal publications, 34 abstracts, and 5 other data sources). Two studies were published as conference abstracts or only within the meta-analysis of several studies (CS35, CS37), one in conference abstracts (149-99-03), and one study as a conference abstract, in FDA safety data publications or within narrative reviews 


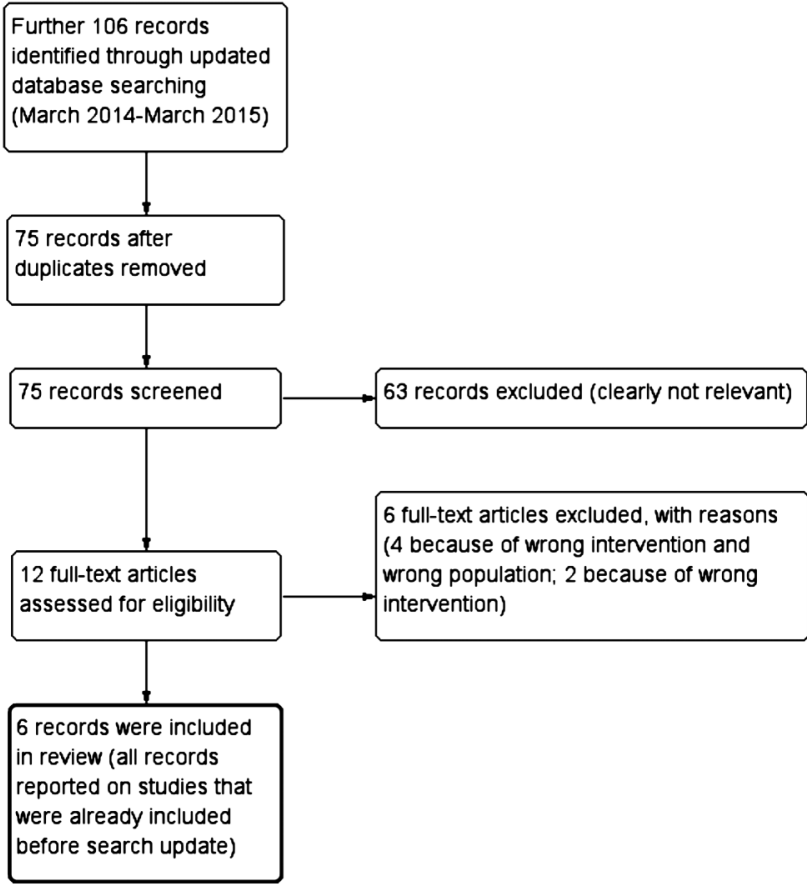

Figure 2 Flow chart of search update in March 2015; adapted to the flow chart recommended by Liberati et al. ${ }^{18}$

(ABACS1). We did not identify journal publications that reported details of the methodology for any of these studies.

We did not identify any active controlled study with follow-up beyond 1 year. There are publications available for an extension of study CS21, which reports on outcomes with longer follow-up. ${ }^{43-76}$ However, randomisation was rescinded in study CS21 after 1 year of follow-up because all patients were switched from GnRH agonist intervention to $\mathrm{GnRH}$ antagonist treatment. Thus, after 1 year of follow-up, this study became an observational study without a control group, and results from this extension phase were not included in this systematic review. Study characteristics of the included studies are presented in tables 2 and 3.

\section{Risk of bias}

Two trials were terminated early (CS28, CS35). Regarding randomised controlled trials, there was adequate information on random sequence generation in only one study (CS21), and on allocation concealment in four studies (CS21, 149-98-02, 149-98-03, 149-99-03). All studies included were open-label trials. Study results for adverse events, treatment failure and quality of life are, therefore, likely to be influenced by lack of blinding. Two studies did not report the dose of GnRH agonist and the number of patients per group included (CS35, CS37). In six studies (CS28, CS31, CS35, CS37, 149-99-03, ABACS1), there was insufficient reporting of attrition and exclusions to permit judgement on incomplete outcome data. One study did not report Gleason score (149-99-03), and four studies did not report either Gleason score or disease stage (ABACS1, 149-97-04, CS35, CS35).

All of the 10 randomised and 3 non-randomised controlled trials provided data on adverse events. However, in five studies, several adverse events were reported incompletely and therefore, could not be entered into our meta-analysis (CS28, CS35, CS37, ABACS1, Zuckerman 2013). There was no wash-out period between the different interventions of the two included cross-over studies (Zuckerman 2013, Garnick 2011).

Details on risk of bias assessment are presented in tables 4-6 and the GRADE evidence profile table (Table 7).

\section{Overall mortality}

Information on mortality presented as time-to-event data was not provided by a single study. Therefore, we could not, as initially planned, analyse these data with HRs, but had to report number of deaths during the study duration. After screening the available entries of the study protocols in the registries, mortality was not predefined as primary or secondary outcome in any of the included studies, but was only assessed as an adverse event outcome.

Nine studies reported the number of patients who had died during study conduct (149-98-02, 149-98-03, ABACS1, CS21, CS28, CS30, CS31, CS35, and CS37). There were no statistically significant differences in deaths between GnRH antagonists and standard androgen suppression therapy (RR $1.35,95 \%$ CI 0.63 to 2.93 , 9 studies with 3020 patients included), nor in the subgroup analyses of abarelix or degarelix compared with standard androgen suppression therapy (abarelix $100 \mathrm{mg}$ : RR 3.49 , 95\% CI 0.77 to $15.83,3$ studies with 697 patients included; degarelix 240/80 and 240/ $160 \mathrm{mg}$ : RR $1.00,95 \%$ CI 0.52 to 1.92 , 6 studies with 2323 patients included; figure 3). Quality of evidence for this outcome was rated low due to study limitations and imprecision according to GRADE (table 7).

\section{PSA progression}

All included studies reported PSA levels, and seven studies reported PSA progression (ABACS1, CS21, CS28, CS30, CS31, CS35 and CS37). Only study CS21 was planned to evaluate time to PSA progression that was defined as two consecutive increases in PSA of $50 \%$ compared with nadir and $\geq 5 \mathrm{ng} / \mathrm{mL}$ on two consecutive measurements, at least 2 weeks apart. ${ }^{37}$ We did not identify a definition for PSA progression for the other studies, and the analyses for PSA progression might be of a post-hoc nature. There was no statistically significant difference in PSA progression between GnRH antagonists and standard androgen suppression therapy (RR 0.83, 95\% CI 0.64 to $1.06,7$ studies with 2489 patients included; subgroup abarelix: RR $1.05,95 \%$ CI 0.41 to 2.66, 1 study with 176 patients included; degarelix 240/80 mg and 240/160 mg: $0.81,95 \%$ CI 0.62 to $1.05,6$ studies with 2313 patients included). We performed post-hoc subgroup analyses for 
Table 2 Study characteristics (degarelix)

\begin{tabular}{|c|c|c|c|c|c|c|c|}
\hline & Zuckerman 2013 & CS21 & CS28 & CS30 & CS31 & CS35 & CS37 \\
\hline $\begin{array}{l}\text { Design } \\
\text { (duration of } \\
\text { study) }\end{array}$ & $\begin{array}{l}\text { Non-randomised } \\
\text { prospective cross-over } \\
\text { study ( } 90 / 90 \text { days) }\end{array}$ & $\begin{array}{l}\text { Randomised } \\
\text { controlled trial } \\
\text { (364 days) }\end{array}$ & $\begin{array}{l}\text { Randomised } \\
\text { controlled trial } \\
\text { (84 days) }\end{array}$ & $\begin{array}{l}\text { Randomised } \\
\text { controlled trial } \\
\text { (84 days) }\end{array}$ & $\begin{array}{l}\text { Randomised } \\
\text { controlled trial } \\
\text { (84 days) }\end{array}$ & $\begin{array}{l}\text { Randomised } \\
\text { controlled trial } \\
\text { (364 days) }\end{array}$ & $\begin{array}{l}\text { Randomised } \\
\text { controlled trial } \\
\text { (364 days) }\end{array}$ \\
\hline $\begin{array}{l}\text { Setting/ } \\
\text { geographical } \\
\text { region }\end{array}$ & Single centre/USA & $\begin{array}{l}\text { Multicentre/ } \\
\text { international }\end{array}$ & Multicentre/Europe & $\begin{array}{l}\text { Multicentre/US, } \\
\text { Europe }\end{array}$ & Multicentre/Europe & $\begin{array}{l}\text { Multicentre/ } \\
\text { international }\end{array}$ & $\begin{array}{l}\text { Multicentre/ } \\
\text { USA }\end{array}$ \\
\hline $\begin{array}{l}\text { Patients } \\
\text { included }\end{array}$ & 48 & 620 & 42 & 246 & 182 & 859 & 405 \\
\hline $\begin{array}{l}\text { Non-metastatic } \\
\text { disease }\end{array}$ & $43(90 \%)$ & $369 / 610(61 \%)$ & 9/40 (22\%) & 235/244 (96\%) & $109 / 179(61 \%)$ & NR & NR \\
\hline $\begin{array}{l}\text { Metastatic } \\
\text { disease }\end{array}$ & $5(10 \%)$ & $125 / 610(20 \%)$ & $14 / 40(35 \%)$ & 0/244 (0\%) & $53 / 179(30 \%)$ & NR & NR \\
\hline $\begin{array}{l}\text { Non-classified } \\
\text { disease }\end{array}$ & - & $116 / 610(19 \%)$ & $17 / 40(43 \%)$ & 9/244 (4\%) & $17 / 179(9 \%)$ & NR & NR \\
\hline $\begin{array}{l}\text { Gleason-Score } \\
2-6\end{array}$ & $9(19 \%)$ & $266 / 610(43 \%)$ & $2 / 40(5 \%)$ & $53 / 244(22 \%)$ & $33 / 179(18 \%)$ & NR & NR \\
\hline $\begin{array}{l}\text { Gleason-Score } \\
7\end{array}$ & $17(35 \%)$ & $181 / 610(30 \%)$ & $38 / 40$ (95\%) & $139 / 244(57 \%)$ & $55 / 179(31 \%)$ & NR & NR \\
\hline $\begin{array}{l}\text { Gleason-Score } \\
8-10\end{array}$ & $22(46)$ & $163 / 610(27 \%)$ & & $52 / 244(21 \%)$ & $91 / 179(51 \%)$ & NR & NR \\
\hline $\begin{array}{l}\text { Gleason-Score } \\
\text { NC }\end{array}$ & - & - & - & - & - & - & - \\
\hline Intervention (N) & $\begin{array}{l}\text { Degarelix } 240 / 80 \mathrm{mg}^{*} \\
(\mathrm{n}=48) \text { for } 3 \text { months }\end{array}$ & $\begin{array}{l}\text { Degarelix } 240 / \\
160 \mathrm{mg}^{\circ} 240 / \\
80 \mathrm{mg}^{*}(\mathrm{n}=409)\end{array}$ & $\begin{array}{l}\text { Degarelix 240/ } \\
80 \mathrm{mg}^{\star}(\mathrm{n}=27)\end{array}$ & $\begin{array}{l}\text { Degarelix 240/ } \\
80 \text { mg* }^{*}(n=181)\end{array}$ & $\begin{array}{l}\text { Degarelix } 240 / \\
80 \mathrm{mg}^{\star}(\mathrm{n}=84)\end{array}$ & $\begin{array}{l}\text { Degarelix } 240 / \\
80 \mathrm{mg}^{\star}(\mathrm{n}=\mathrm{NR})\end{array}$ & $\begin{array}{l}\text { Degarelix 240/ } \\
80 \mathrm{mg}^{\star}(\mathrm{n}=\mathrm{NR})\end{array}$ \\
\hline Control (N) & $\begin{array}{l}\text { Leuprolide (22.5 mg) } \\
\text { 3-month depot for } \\
\text { 3-month }\end{array}$ & $\begin{array}{l}\text { Leuprolide } 7.5 \mathrm{mg} \\
(\mathrm{n}=201) \text { monthly }\end{array}$ & $\begin{array}{l}\text { Goserelin } 3.6 \mathrm{mg} \\
\text { monthly }+ \\
\text { bicalutamide } 50 \mathrm{mg} \\
\text { daily }(n=13)\end{array}$ & $\begin{array}{l}\text { Goserelin } 3.6 \text { mg } \\
\text { monthly + } \\
\text { bicalutamide } 50 \text { mg } \\
\text { daily }(n=65)\end{array}$ & $\begin{array}{l}\text { Goserelin } 3.6 \mathrm{mg} \\
\text { monthly }+ \\
\text { bicalutamide } 50 \mathrm{mg} \\
\text { daily }(\mathrm{n}=98)\end{array}$ & $\begin{array}{l}\text { Goserelin NR } \\
m g(n=N R)\end{array}$ & $\begin{array}{l}\text { Leuprolide NR } \\
\mathrm{mg}(\mathrm{n}=\mathrm{NR})\end{array}$ \\
\hline Outcomes & $\begin{array}{l}\text { Ability to maintain } \\
\text { medical castration } \\
\text { (prevent a testosterone } \\
\text { surge) during transition } \\
\text { from degarelix to } \\
\text { leuprolide, assessment } \\
\text { of any PSA elevation } \\
\text { after the degarelix to } \\
\text { leuprolide transition, } \\
\text { adverse events }\end{array}$ & $\begin{array}{l}\text { Change in vital } \\
\text { signs/body weight/ } \\
\text { QTc Interval, } \\
\text { adverse events, } \\
\text { measurement of } \\
\text { PSA levels/ } \\
\text { testosterone levels/ } \\
\text { testosterone surge, } \\
\text { time to PSA failure }\end{array}$ & $\begin{array}{l}\text { Change in vital } \\
\text { signs/body weight/ } \\
\text { total IPSS/quality of } \\
\text { life/prostate size/ } \\
\text { maximum urine flow/ } \\
\text { residual volume, } \\
\text { measurement of } \\
\text { PSA levels/ } \\
\text { testosterone levels, } \\
\text { adverse events }\end{array}$ & $\begin{array}{l}\text { Change in vital } \\
\text { signs and body } \\
\text { weight/laboratory } \\
\text { variables/oestradiole } \\
\text { levels/total IPSS/ } \\
\text { quality of life/ } \\
\text { prostate size, } \\
\text { measurement of } \\
\text { PSA levels/ } \\
\text { testosterone levels, } \\
\text { adverse events }\end{array}$ & $\begin{array}{l}\text { Change in vital } \\
\text { signs/body weight/ } \\
\text { laboratory variables/ } \\
\text { total IPSS/ quality of } \\
\text { life/benign prostatic } \\
\text { hyperplasia impact } \\
\text { index/prostate size, } \\
\text { measurement of } \\
\text { PSA levels/ } \\
\text { testosterone levels, } \\
\text { adverse events }\end{array}$ & $\begin{array}{l}\text { Change in total } \\
\text { IPSS/quality of } \\
\text { life, } \\
\text { measurement of } \\
\text { PSA levels/ } \\
\text { testosterone } \\
\text { levels }\end{array}$ & $\begin{array}{l}\text { Measurement } \\
\text { of PSA levels, } \\
\text { change in } \\
\text { quality of life }\end{array}$ \\
\hline
\end{tabular}

. $240 \mathrm{mg}$ subcutaneous given as a starting dose and $80 \mathrm{mg}$ or $160 \mathrm{mg}$ subcutaneous maintenance doses every 4 weeks, thereafter.

IPSS, International Prostate Symptom Score; NC, not classified; NR, not reported; PSA, prostate-specific antigen. 


\begin{tabular}{|c|c|c|c|c|c|c|}
\hline & 149-98-02 & $149-98-03$ & $149-99-03$ & ABACS 1 & $149-97-04$ & Garnick 2011 \\
\hline $\begin{array}{l}\text { Design (duration } \\
\text { of study) }\end{array}$ & $\begin{array}{l}\text { Randomised controlled } \\
\text { trial ( } 169 \text { days) }\end{array}$ & $\begin{array}{l}\text { Randomised controlled } \\
\text { trial ( } 169 \text { days) }\end{array}$ & $\begin{array}{l}\text { Randomised controlled } \\
\text { trial ( } 169 \text { days) }\end{array}$ & $\begin{array}{l}\text { Randomised } \\
\text { controlled trial } \\
\text { (364 days) }\end{array}$ & $\begin{array}{l}\text { Prospective } \\
\text { non-randomised } \\
\text { controlled clinical trial } \\
\text { ( } 27 \text { days) }\end{array}$ & $\begin{array}{l}\text { Non-randomised } \\
\text { prospective cross-over } \\
\text { study ( } 84 / 56 \text { days) }\end{array}$ \\
\hline $\begin{array}{l}\text { Geographical } \\
\text { region }\end{array}$ & Multicentre/USA & Multicentre/USA & Multicentre/USA & Multicentre/Europe & Multicentre/USA & Multicentre/USA \\
\hline $\begin{array}{l}\text { Patients } \\
\text { included }\end{array}$ & 271 & 255 & 584 & 177 & 242 & 176 \\
\hline $\begin{array}{l}\text { Non-metastatic } \\
\text { disease }\end{array}$ & $165 / 269(61 \%)$ & $145 / 251(58 \%)$ & NR & NR & NR & $143 / 176(80 \%)$ \\
\hline $\begin{array}{l}\text { Metastatic } \\
\text { disease }\end{array}$ & 104/269 (39\%) & $106 / 251(42 \%)$ & $30 / 582(5 \%)$ & NR & NR & $12 / 176(8 \%)$ \\
\hline $\begin{array}{l}\text { Non-classified } \\
\text { disease }\end{array}$ & - & - & $552 / 582(95 \%)$ & - & - & $21 / 176(12 \%)$ \\
\hline $\begin{array}{l}\text { Gleason-Score } \\
2-6\end{array}$ & $121 / 269(45 \%)$ & $144 / 251(57 \%)$ & NR & NR & NR & $97 / 176(55 \%)$ \\
\hline Gleason-Score 7 & $81 / 269$ (30\%) & $61 / 251(24 \%)$ & NR & NR & NR & 73/176 (41\%) \\
\hline $\begin{array}{l}\text { Gleason-Score } \\
8-10\end{array}$ & $56 / 269$ (21\%) & $34 / 251(14 \%)$ & NR & NR & NR & $6 / 176(3 \%)$ \\
\hline $\begin{array}{l}\text { Gleason-Score } \\
\text { non-classified }\end{array}$ & $11 / 269(4 \%)$ & $12 / 251(5 \%)$ & - & - & - & - \\
\hline Intervention (N) & $\begin{array}{l}\text { Abarelix } 100 \mathrm{mg}^{*} \\
(\mathrm{n}=180)\end{array}$ & $\begin{array}{l}\text { Abarelix } 100 \mathrm{mg}^{*} \\
(n=170)\end{array}$ & $\begin{array}{l}\text { Abarelix } 100 \mathrm{mg}^{*} \\
(\mathrm{n}=390)\end{array}$ & $\begin{array}{l}\text { Abarelix } 100 \mathrm{mg}^{*} \\
(\mathrm{n}=87)\end{array}$ & $\begin{array}{l}\text { Abarelix } 100 \mathrm{mg}^{*} \\
(\mathrm{n}=209)\end{array}$ & $\begin{array}{l}\text { Abarelix } 100 \mathrm{mg}^{*} \\
(\mathrm{n}=176)\end{array}$ \\
\hline Control (N) & $\begin{array}{l}\text { Leuprolide } 7.5 \mathrm{mg} \\
\text { monthly }(\mathrm{n}=91)\end{array}$ & $\begin{array}{l}\text { Leuprolide } 7.5 \mathrm{mg} \\
\text { monthly + bicalutamide } \\
50 \text { mg daily }(n=85)\end{array}$ & $\begin{array}{l}\text { Leuprolide } 7.5 \mathrm{mg} \\
\text { monthly }(\mathrm{n}=194)\end{array}$ & $\begin{array}{l}\text { Goserelin } 3.6 \mathrm{mg} \\
\text { monthly }+ \\
\text { bicalutamide } 50 \mathrm{mg} \\
\text { daily }(n=90)\end{array}$ & $\begin{array}{l}\text { Leuprolide or Goserelin } \\
\text { with(out) antiandrogen } \\
(n=33)\end{array}$ & $\begin{array}{l}\text { Leuprolide } 7.5 \mathrm{mg} \\
\text { monthly or goserelin } \\
3.6 \mathrm{mg} \text { monthly } \\
(\mathrm{n}=176)\end{array}$ \\
\hline Outcomes & $\begin{array}{l}\text { Achievement of } \\
\text { castration (day }<8,<29 \text {, } \\
<365 \text { ); measurement of } \\
\text { testosterone levels/ } \\
\text { endocrine efficacy/PSA } \\
\text { levels, adverse events }\end{array}$ & $\begin{array}{l}\text { Achievement of } \\
\text { castration (day }<8,<29 \text {, } \\
<365 \text { ); measurement of } \\
\text { testosterone levels/ } \\
\text { endocrine efficacy/PSA } \\
\text { levels, adverse events }\end{array}$ & $\begin{array}{l}\text { Achievement of } \\
\text { castration (day }<8 \text {, } \\
<365 \text { ); adverse events, } \\
\text { discontinuation of } \\
\text { treatment, } \\
\text { measurement of PSA } \\
\text { levels }\end{array}$ & $\begin{array}{l}\text { Achievement of } \\
\text { castration (day <8, } \\
<365 \text { ), } \\
\text { measurement of } \\
\text { testosterone levels, } \\
\text { adverse events }\end{array}$ & $\begin{array}{l}\text { Achievement of } \\
\text { castration (day }<8 \text {, } \\
<365 \text { ), measurement of } \\
\text { testosterone levels/ } \\
\text { endocrine efficacy/PSA } \\
\text { levels, adverse events }\end{array}$ & $\begin{array}{l}\text { Achievement of } \\
\text { castration (day }<8 \text {, } \\
<365 \text { ), measurement of } \\
\text { testosterone levels, } \\
\text { adverse events }\end{array}$ \\
\hline
\end{tabular}


Table 4 Risk of bias assessment per randomised controlled trial (degarelix)

\begin{tabular}{|c|c|c|c|c|c|c|}
\hline Random sequence generation & Low risk* & Unclear risk (NR) & Unclear risk (NR) & Unclear risk (NR) & Unclear risk (NR) & Unclear risk (NR) \\
\hline Allocation concealment & Low risk† & Unclear risk (NR) & Unclear risk (NR) & Unclear risk (NR) & Unclear risk (NR) & Unclear risk (NR) \\
\hline $\begin{array}{l}\text { Blinding of participants and personnel: mortality, PSA } \\
\text { progression }\end{array}$ & Low risk & Unclear risk (NR) & Low risk $\ddagger$ & Unclear risk (NR) & Unclear risk (NR) & Unclear risk (NR) \\
\hline $\begin{array}{l}\text { Blinding of participants and personnel: adverse events, } \\
\text { treatment failure, quality of life }\end{array}$ & High risk§ & High risk§ & High risk§ & High risk§ & High risk§ & High risk§ \\
\hline $\begin{array}{l}\text { Blinding of outcome assessment: Mortality, PSA } \\
\text { progression }\end{array}$ & Low riskł & Unclear risk (NR) & Low risk $\ddagger$ & Unclear risk (NR) & Unclear risk (NR) & Unclear risk (NR) \\
\hline $\begin{array}{l}\text { Blinding of outcome assessment: Adverse events, treatment } \\
\text { failure, quality of life }\end{array}$ & High risk§ & High risk§ & High risk§ & High risk§ & High risk§ & High risk§ \\
\hline Incomplete outcome data: mortality, PSA progression & Low riskף & Unclear risk (NR) & Low riskף & Unclear risk (NR) & Unclear risk (NR) & Unclear risk (NR) \\
\hline $\begin{array}{l}\text { Incomplete outcome data: adverse events, treatment failure, } \\
\text { quality of life }\end{array}$ & Low riskๆ & Unclear risk (NR) & Low riskף & Unclear risk (NR) & Unclear risk (NR) & Unclear risk (NR) \\
\hline Selective reporting & Low risk ${ }^{* *}$ & High risk†† & Low risk ${ }^{\star *}$ & Low risk ${ }^{* *}$ & High risk†† & High risk†† \\
\hline \multicolumn{7}{|c|}{ 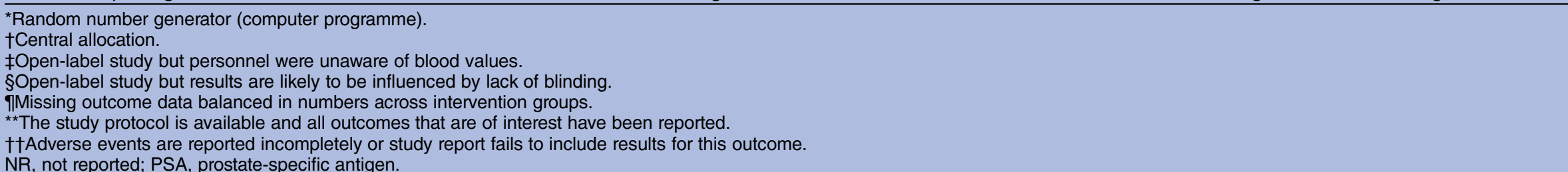 } \\
\hline
\end{tabular}


Table 5 Risk of bias assessment per randomised controlled trial (abarelix)

\begin{tabular}{|c|c|c|c|c|}
\hline & $149-98-02$ & $149-98-03$ & $149-99-03$ & ABACS1 \\
\hline Random sequence generation & Unclear risk (NR) & Unclear risk (NR) & Unclear risk (NR) & Unclear risk (NR) \\
\hline Allocation concealment & Low risk* & Low risk* & Low risk ${ }^{*}$ & Unclear risk (NR) \\
\hline $\begin{array}{l}\text { Blinding of participants and personnel: } \\
\text { mortality, PSA progression }\end{array}$ & Low risk† & Low risk† & Unclear risk (NR) & Unclear risk (NR) \\
\hline $\begin{array}{l}\text { Blinding of participants and personnel: adverse } \\
\text { events, treatment failure, quality of life }\end{array}$ & High risk $\ddagger$ & High risk $\ddagger$ & High risk $\ddagger$ & High risk $\ddagger$ \\
\hline $\begin{array}{l}\text { Blinding of outcome assessment: mortality, } \\
\text { PSA progression }\end{array}$ & Low risk† & Low risk† & Unclear risk (NR) & Unclear risk (NR) \\
\hline $\begin{array}{l}\text { Blinding of outcome assessment: adverse } \\
\text { events, treatment failure, quality of life }\end{array}$ & High risk $\ddagger$ & High risk $\ddagger$ & High risk $\ddagger$ & High risk‡ \\
\hline $\begin{array}{l}\text { Incomplete outcome data: mortality, PSA } \\
\text { progression }\end{array}$ & Low risk§ & Low risk§ & Unclear risk (NR) & Unclear risk (NR) \\
\hline $\begin{array}{l}\text { Incomplete outcome data: adverse events, } \\
\text { treatment failure, quality of life }\end{array}$ & Low risk§ & Low risk§ף & Unclear risk (NR) & Unclear risk (NR) \\
\hline Selective reporting & Low risk ${ }^{\star \star}$ & Low risk ${ }^{\star \star}$ & Unclear risk $\dagger \dagger$ & High riskł‡ \\
\hline \multicolumn{5}{|c|}{$\begin{array}{l}\text { *Central allocation. } \\
\text { tOpen-label study but personnel were unaware of blood values. } \\
\text { tOpen-label study but results are likely to be influenced by lack of blinding. } \\
\text { §Proportion of missing outcomes compared with observed event risk not enough to have a clinically relevant impact on the intervention effect } \\
\text { estimate. } \\
\text { qMissing outcome data balanced in numbers across intervention groups. } \\
\text { †The study protocol is not available but it is clear that the published reports include all expected outcomes. } \\
\text { t†No protocol available. } \\
\text { tłAdverse events are reported incompletely or study report fails to include results for this outcome. } \\
\text { NR, not reported; PSA, prostate-specific antigen. }\end{array}$} \\
\hline
\end{tabular}

patients treated with degarelix and different baseline PSA levels. There were no statistically significant differences for patients treated with different regimens of degarelix, that is, $240 / 80 \mathrm{mg}$ or $240 / 160 \mathrm{mg}$ and PSA $\leq 50 \mathrm{ng} / \mathrm{mL}(\mathrm{PSA}<20 \mathrm{ng} / \mathrm{mL}: \mathrm{RR} 9.10,95 \%$ CI 0.52 to 159.00 , 6 studies with 1399 patients included; PSA $\geq 20$ $50 \mathrm{ng} / \mathrm{mL}$ : RR $0.81,95 \%$ CI 0.34 to $1.90,6$ studies with 401 patients included). GnRH antagonists decreased PSA progression in patients with baseline PSA levels $>50 \mathrm{ng} /$ $\mathrm{mL}$ compared with standard androgen suppression therapy (RR $0.74,95 \%$ CI 0.56 to $0.98,6$ studies with 513 patients included). Quality of evidence was rated low due to study limitations and imprecision according to GRADE (table 7).

\section{Treatment failure}

Seven studies reported treatment failure (149-98-02, 149-98-03, 149-99-03, CS21, CS28, CS30 and CS31). No statistically significant differences were observed between GnRH antagonists and standard androgen suppression therapy (RR $0.91,95 \%$ CI 0.70 to $1.17,77$ studies with 2200 patients included). While subgroup analyses demonstrated a favourable effect for abarelix compared with standard androgen suppression therapy (RR $0.66,95 \%$ CI 0.45 to $0.98,3$ studies with 1110 patients included), there was no significant difference for degarelix compared with standard therapy (degarelix 240/80 mg: RR $1.03,95 \%$ CI 0.65 to 1.63 , 4 studies with 782 patients included; degarelix 240/160 mg: RR $1.33,95 \%$ CI 0.79 to $2.24,1$ study with 308 patients included). Quality of evidence was rated low due to study limitations and imprecision according to GRADE (table 7).

At variance with the prespecified outcomes in our protocol, we also included the outcome 'failure to achieve or maintain castration'. Castration was defined as no testosterone value $>50 \mathrm{ng} / \mathrm{mL}$ under androgen suppression therapy. Five studies provided data (149-98-02, 149-98-03, 149-99-03, ABACS1, and CS21). We identified a statistically significant difference in favour of standard androgen suppression therapy (RR $1.80,95 \%$ CI 1.37 to $2.35,5$ studies with 1889 patients included). However, statistically significant differences did not persist after using the random effects model for heterogeneity $\left(\mathrm{I}^{2}=60 \%\right.$; RR $1.53,95 \%$ CI 0.95 to $2.49,5$ studies with 1889 patients included). Therefore, the overall effect on this outcome remains unclear. Subgroup analyses showed that abarelix increased the failure to achieve or maintain castration, while there was no significant difference between degarelix and standard therapy (abarelix: RR 1.88, 95\% CI 1.19 to $2.97 ; 4$ studies with 1279 patients included; degarelix 240/ 80 mg: RR $0.61,95 \%$ CI 0.17 to 2.22 , 1 study with 308 patients included; degarelix 240/160 mg: RR 0.50, 95\% CI 0.10 to $2.41,1$ study with 302 patients included).

\section{Adverse events}

The data on adverse events are shown in table 8. We did not identify statistically significant differences for the predefined adverse events fatigue, hot flushes, infections, loss of sexual interest, sexual dysfunction, asthenia, urinary retention, diarrhoea, or constipation (table 8). 
Table 6 Risk of bias assessment per prospective non-randomised comparator controlled studies (degarelix + abarelix) ${ }^{*}$

\begin{tabular}{llll}
\hline & $\mathbf{1 4 9 - 9 7 - 0 4}$ & Zuckerman 2013 & Garnick 2011 \\
\hline Study type & controlled clinical trial & cross-over study & cross-over study \\
Prospective study? & Yes & Yes & Yes \\
Was there a comparison? & Yes & Yes & Yes \\
Was there a baseline assessment? & Yes & Yes & Yes \\
Blinding of outcome assessment? & Unclear & No & No \\
Incomplete outcome data? & Yes & No & No \\
Selective outcome reporting? & Unclear & & Unclear \\
Patient selection method & No & No & No \\
Random sample generation & Yes & Unclear & Yes \\
Consecutive enrolment & Yes & Unclear & No \\
Selected subset of patients & No & No & No \\
Time difference & No & No & No \\
Location difference & Yes & No & No \\
Treatment decision & Yes & No & No \\
Patients preferences & No & Unclear & No \\
On the basis of outcome & Unclear & No & Unclear \\
Predefinition of adverse events? & Unclear & Yes & Unclear \\
Reporting of all adverse events? & Unclear & No & Unclear \\
Are all patients evaluated for adverse events? & Unclear & Unclear \\
Dropouts because of adverse events? & *Adapted to the checklist recommended by Reeves et al for data collection and study assessment for non-randomised studies
\end{tabular}

The risk of injection site pain or reaction significantly increased with GnRH antagonists compared with standard therapy (table 8).

No significant difference in urinary tract infection was observed between the different therapy groups. However, subgroup analysis showed a significant positive effect for degarelix $240 / 80$ or $240 / 160 \mathrm{mg}$ compared with standard androgen therapy (RR 0.57 ; $95 \%$ CI 0.39 to $0.83,6$ studies with 2328 patients included; table 8 ).

Cardiovascular events occurred less often with GnRH antagonist (degarelix 240/80 and 240/160 mg) than with standard therapy (RR $0.60,95 \%$ CI 0.38 to 0.94, 6 studies with 2328 patients included; table 8). Given the reduced risk regarding cardiovascular events, we also evaluated further adverse events regarding the cardiovascular system. Post hoc analyses revealed no statistically significant differences regarding acute myocardial infarction or fatal cerebrovascular-related events, but showed that new diagnosis of ischaemic heart diseases occurred significantly less often in patients who were using GnRH antagonists compared with patients on standard androgen suppression therapy (RR $0.42,95 \%$ CI 0.23 to $0.77,1$ study with 610 patients included). This was also seen for the subgroup of patients treated with degarelix $240 / 80 \mathrm{mg}$, but not for those treated with degarelix $240 / 160 \mathrm{mg}$. Therefore, the effect of GnRH antagonists on these post hoc included outcomes remains unclear. Additionally, it was also unclear if these results are also applicable for patients who already had a history of cardiovascular events because original publications did not report if this was evaluated during the study screening phase or if this was an exclusion criteria.
The risks of experiencing peripheral oedema and musculoskeletal adverse events were decreased using GnRH antagonists compared with standard androgen suppression therapy (RR $0.51,95 \%$ CI 0.32 to $0.81,2$ studies with 520 patients included and RR 0.65 , 95\% CI 0.45 to $0.96,1$ study with 408 patients included, respectively).

Arthralgia and back pain also occurred less often with GnRH antagonists (table 8). However, this was only seen in the subgroup of patients treated with degarelix (RR $0.66,95 \%$ CI 0.46 to $0.94,6$ studies with 2328 patients included, and RR $0.68,95 \%$ CI 0.48 to $0.99,6$ studies with 2328 patients included, respectively).

Meta-analysis identified that the risk of chills was increased with GnRH antagonists (RR 9.38, 95\% CI 1.26 to $69.58,1$ study with 610 patients included). Interestingly, no chills occurred with standard androgen suppression therapy (18/409 degarelix vs 0/201 standard androgen suppression therapy).

There were no statistically significant differences regarding serious adverse events (RR 0.82, 95\% CI 0.62 to $1.08,7$ studies with 2179 patients included), severe/ life-threatening adverse events (RR $0.76,95 \%$ CI 0.58 to $1.00,5$ studies with 2064 patients included), or discontinuations due to adverse events (RR $0.86,95 \%$ CI 0.57 to 1.31, 8 studies with 2290 patients included).

We identified no statistical significant differences between GnRH antagonists and standard androgen suppression therapy for immediate-onset allergic reactions (RR 2.36, 95\% CI 0.55 to $10.12,5$ studies with 1694 patients included, table 8). However, this adverse event occurred in 9 of 1119 patients $(0.8 \%)$ treated with abarelix but in no patient receiving standard androgen 
Table 7 GRADE evidence table: quality of evidence assessment (confidence in effect estimates) per end point

\begin{tabular}{|c|c|c|c|c|c|c|c|c|c|c|c|}
\hline \multicolumn{7}{|c|}{ Quality assessment } & \multicolumn{2}{|l|}{ Patients (n) } & \multicolumn{2}{|l|}{ Effect } & \multirow[b]{2}{*}{ Quality } \\
\hline $\begin{array}{l}\text { No of } \\
\text { studies }\end{array}$ & Design & $\begin{array}{l}\text { Risk of } \\
\text { bias }\end{array}$ & Inconsistency & Indirectness & Imprecision & $\begin{array}{l}\text { Other } \\
\text { considerations }\end{array}$ & $\begin{array}{l}\text { GnRH } \\
\text { antagonists }\end{array}$ & $\begin{array}{l}\text { Standard } \\
\text { androgen } \\
\text { suppression } \\
\text { therapy }\end{array}$ & $\begin{array}{l}\text { Relative } \\
(95 \% \mathrm{Cl}) \\
\end{array}$ & Absolute & \\
\hline \multicolumn{12}{|c|}{ Overall mortality (follow-up 84-364 days) } \\
\hline 9 & $\begin{array}{l}\text { Randomised } \\
\text { trials* }\end{array}$ & Serioust & $\begin{array}{l}\text { No serious } \\
\text { inconsistency }\end{array}$ & $\begin{array}{l}\text { No serious } \\
\text { indirectness }\end{array}$ & Serious $\ddagger$ & See comment§ & $\begin{array}{l}35 / 1923 \\
(1.8 \%)\end{array}$ & $\begin{array}{l}16 / 1097 \\
(1.5 \%)\end{array}$ & $\begin{array}{l}\text { RR } 1.35 \\
(0.63 \text { to } 2.93)\end{array}$ & $\begin{array}{l}5 \text { more per } 1000 \\
\text { (from } 6 \text { fewer to } 30 \\
\text { more) }\end{array}$ & $\begin{array}{l}\oplus \oplus \mathrm{OO} \\
\text { LOW }\end{array}$ \\
\hline \multicolumn{12}{|c|}{ Treatment failure (follow-up 84-364 days) } \\
\hline 7 & $\begin{array}{l}\text { Randomised } \\
\text { trials } \|\end{array}$ & Serious ${ }^{\star *}$ & $\begin{array}{l}\text { No serious } \\
\text { inconsistency }\end{array}$ & $\begin{array}{l}\text { No serious } \\
\text { indirectness }\end{array}$ & Serious $\ddagger$ & None & $\begin{array}{l}146 / 1450 \\
(10.1 \%)\end{array}$ & $\begin{array}{l}81 / 750 \\
(10.8 \%)\end{array}$ & $\begin{array}{l}\text { RR } 0.92 \\
\text { (0.64 to } 1.33)\end{array}$ & $\begin{array}{l}9 \text { fewer per } 1000 \\
\text { (from } 39 \text { fewer to } \\
36 \text { more) }\end{array}$ & $\begin{array}{l}\oplus \oplus \mathrm{OO} \\
\mathrm{LOW}\end{array}$ \\
\hline \multicolumn{12}{|c|}{ PSA progression (follow-up 84-364 days) } \\
\hline 7 & $\begin{array}{l}\text { Randomised } \\
\text { trialst† }\end{array}$ & Seriousł‡ & $\begin{array}{l}\text { No serious } \\
\text { inconsistency }\end{array}$ & $\begin{array}{l}\text { No serious } \\
\text { indirectness }\end{array}$ & Serious $\ddagger$ & None & $\begin{array}{l}115 / 1566 \\
(7.3 \%)\end{array}$ & $\begin{array}{l}75 / 923 \\
(8.1 \%)\end{array}$ & $\begin{array}{l}\text { RR } 0.83 \\
\text { (0.64 to } 1.06)\end{array}$ & $\begin{array}{l}14 \text { fewer per } 1000 \\
\text { (from } 29 \text { fewer to } 5 \\
\text { more) }\end{array}$ & $\begin{array}{l}\oplus \oplus \mathrm{OO} \\
\mathrm{LOW}\end{array}$ \\
\hline \multicolumn{12}{|c|}{ Quality of life related to IPSS, follow-up 84 days; better indicated by lower values } \\
\hline 3 & $\begin{array}{l}\text { Randomised } \\
\text { trials§§ }\end{array}$ & Serious ๆी & $\begin{array}{l}\text { No serious } \\
\text { inconsistency }\end{array}$ & Serious ${ }^{\star \star \star}$ & $\begin{array}{l}\text { No serious } \\
\text { imprecision }\end{array}$ & None & 286 & 173 & - & $\begin{array}{l}\text { MD } 1.84 \text { lower ( } 3 \text { to } \\
0.69 \text { lower) }\end{array}$ & $\begin{array}{l}\oplus \oplus \mathrm{OO} \\
\text { LOW }\end{array}$ \\
\hline \multicolumn{12}{|c|}{ Quality of life related to urinary symptoms (follow-up 84 days; better indicated by lower values) } \\
\hline 3 & $\begin{array}{l}\text { Randomised } \\
\text { trials§§ }\end{array}$ & Seriousףी & $\begin{array}{l}\text { No serious } \\
\text { inconsistency }\end{array}$ & Serious $^{\star \star *}$ & $\begin{array}{l}\text { No serious } \\
\text { imprecision }\end{array}$ & None & 288 & 173 & - & $\begin{array}{l}\text { MD } 0.4 \text { lower ( } 0.94 \\
\text { lower to } 0.14 \\
\text { higher) }\end{array}$ & $\begin{array}{l}\oplus \oplus \mathrm{OO} \\
\mathrm{LOW}\end{array}$ \\
\hline
\end{tabular}

*The following studies were included: 149-98-02, 149-98-03, ABACS1, CS21, CS28, CS30, CS31, CS35, CS37.

†Downgraded for study limitations (-1): High or unclear risk of bias in included studies (for details see 'risk of bias' tables). Despite the methodological limitations, we do not feel that results are likely to be influenced by lack of blinding. However, there was insufficient reporting of attrition and exclusions to permit judgment on incomplete outcome data in studies CS28, CS31, CS35,

CS37, and ABACS1. Studies CS35 and CS37 were reported as conference abstracts or data presentation within combined data analyses. Study ABACS1 was reported as conference abstract

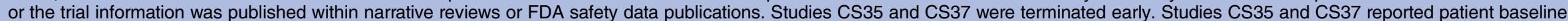
characteristics incompletely.

fDowngraded for imprecision (-1): Imprecision due to low number of events and wide Cls.

§Information on mortality was not provided by a single study as time-to-event data. Therefore, we could not, as initially planned, analyse these data with HRs, but have to report number of deaths during study duration. After screening the available entries of the study protocols in the registries, mortality was not predefined as primary/secondary outcome in any of the included studies, but was only assessed as an adverse event outcome.

१The following studies were included: 149-98-02, 149-98-03, 149-99-03, CS21, CS28, CS30, CS31.

${ }_{\star \star *}$ Downgraded for study limitations (-1): High or unclear risk of bias in included studies (for details see 'risk of bias' tables). Study $149-99-03$ was reported as conference abstract only. There was insufficient reporting of attrition and exclusions to permit judgment on incomplete outcome data in studies CS28, CS31, and 149-99-03. Study CS28 was terminated early.

t†The following studies were included: CS21, CS28, CS30, CS31, CS35, CS37, ABACS1.

fłDowngraded for study limitations (-1): High or unclear risk of bias in included studies (for details see 'risk of bias' tables). Despite the methodological limitations, we do not feel that results are likely to be influenced by lack of blinding. However, there was insufficient reporting of attrition and exclusions to permit judgment on incomplete outcome data in studies CS28, CS31, CS35, CS37, and ABACS1. Studies CS35 and CS37 were reported as conference abstracts or data presentation within combined data analyses only. Study ABACS1 was reported as conference

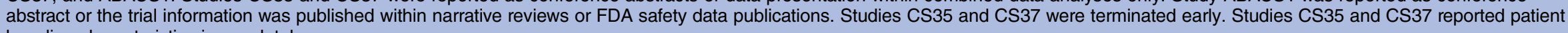
baseline characteristics incompletely.

$\S \S$ The following studies were included: CS28, CS30, CS31.

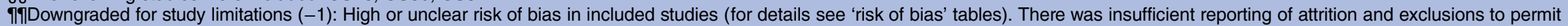
judgment on incomplete outcome data in studies CS28 and CS31. Studies CS35 and CS37 were identified to measure quality of life outcomes. However, we found no publications of these studies that reported this outcome.

${ }_{* * *}$ Downgraded for indirectness $(-1)$ : The question addressed by this systematic review was different from the results presented in the available evidence. We expected a measurement of quality of life related to general health but found only an evaluation of quality of life related to urinary symptoms or IPSS.

FDA, Food and Drug Administration; GnRH, gonadotropin-releasing hormone; IPSS, International Prostate Symptom Score; MD, mean difference. 


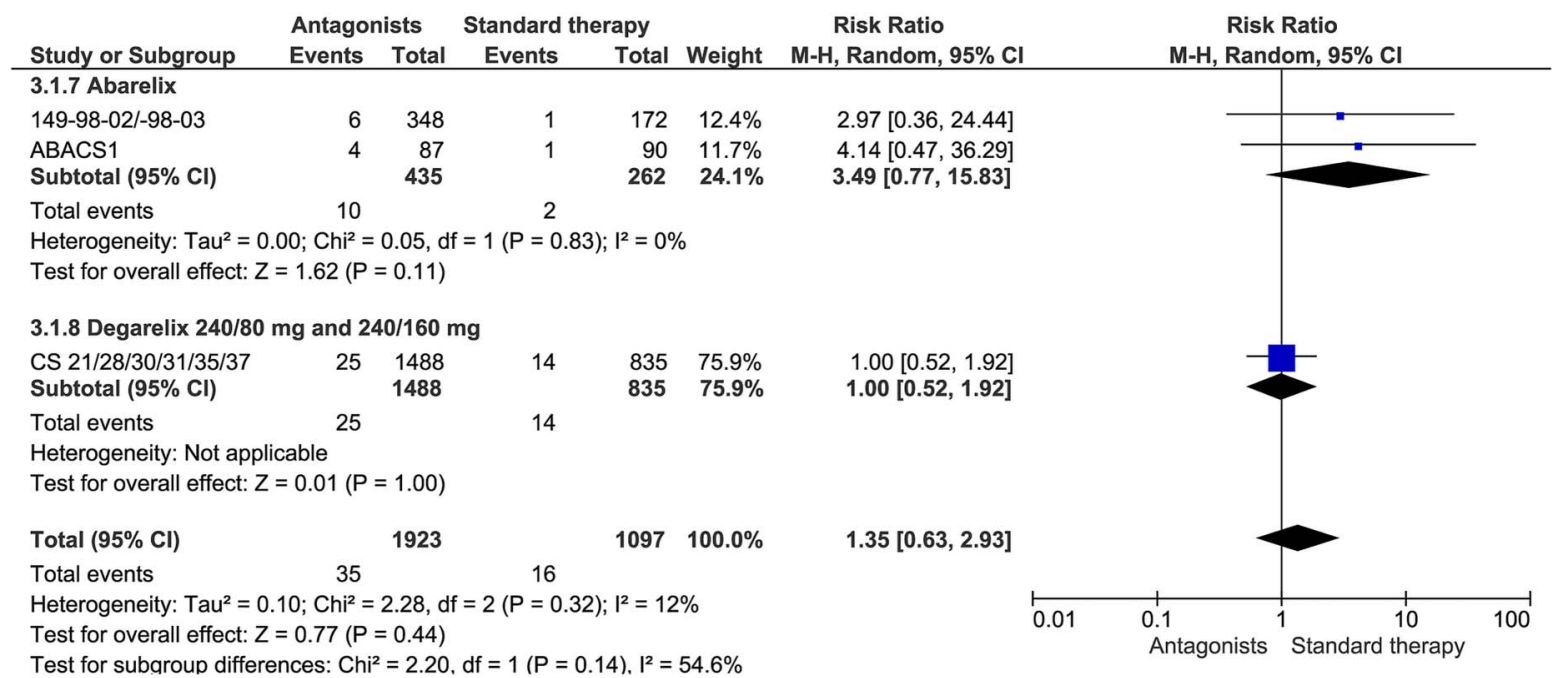

Figure 3 Overall mortality.

suppression therapy. We found no data for degarelix regarding this outcome.

We did not identify information about the occurrence of gynaecomastia, breast pain or sweating with the use of GnRH antagonist therapy.

\section{Quality of life}

Three studies were included for quality of life evaluation (CS28, CS20, and CS31). Further two studies (CS35 and CS37) were identified to measure quality of life outcomes through screening of protocol entries. However, we found no publications of these studies that reported this outcome. The question addressed by this systematic review was different from the results presented in included studies because we expected a measurement of quality of life related to general health, but instead found an evaluation of quality of life related to urinary or prostate symptoms only.

While there was no statistically significant difference for quality of life related to urinary symptoms, improved quality of life regarding prostate symptoms measured with the International Prostate Symptom Score (IPSS) with the use of GnRH antagonists (degarelix 240/ $80 \mathrm{mg}$ ) compared with the use of standard androgen suppression therapy (mean score difference $-0.40,95 \%$ CI -0.94 to $0.14,3$ studies with 461 patients included, and $-1.84,95 \%$ CI -3.00 to $-0.69,3$ studies with 459 patients included, respectively) was found. Quality of evidence was rated low according to GRADE (table 7).

\section{DISCUSSION}

Based on the assessed evidence, including trials not published as journal articles, the effects on efficacy of GnRH antagonist compared to standard androgen therapy are still unclear since no long-term follow-up data (>364 days) are available for any of the evaluated outcomes and as evidence is hampered by selective reporting of results, risk of bias, and insufficient reporting of methodology. Fifteen studies were identified, but only 13 could be included. No study reported cancer-specific survival or clinical progression. There were no statistically significant differences in overall mortality, treatment failure, PSA progression or quality of life. However, quality of evidence according to GRADE was rated low for these outcomes.

The question addressed by this systematic review could partly not be answered with the available evidence. We aimed to assess efficacy and safety of GnRH antagonists compared with standard androgen suppression therapy for advanced prostate cancer treatment. However, most of the studies available were not intended to provide, as their primary end point, safety and efficacy data. The majority of studies included were performed or sponsored by the manufacturing companies to gain regulatory approval for marketing authorisation. The studies aimed to assess the pharmacodynamic metrics of obtaining a level of testosterone $</=50 \mathrm{ng} / \mathrm{dL}$ by day 28 , and maintaining that level through 365 days. The primary outcome of two studies (CS30 and CS31) was the evaluation of prostate volume reduction and relief from lower urinary tract symptoms. In one study (CS21), many patients had localised disease or PSA relapse only. The majority of patients treated with androgen suppression therapy for prostate cancer had non-metastatic disease (range 58-96\%), and the number of patients with Gleason score $<7$ ranged between $18 \%$ (CS31) and $57 \%$ (149-98-03). Future studies, therefore, should focus on patient-relevant outcomes to inform decision-making in clinical practice.

The FDA required a black-box warning on the packaging and the patient instruction sheet of abarelix in USA because immediate-onset systemic allergic reactions occurred after administration of this drug. We found no statistically significant differences in immediate-onset allergic reactions between GnRH antagonists and standard androgen suppression therapy. However, it should be mentioned that $1.1 \%$ of patients included in FDA 
Table 8 Adverse events

\section{Outcome or subgroup}

Serious adverse events

Subgroup: abarelix $100 \mathrm{mg}$

Subgroup: degarelix 240/160 mg

Subgroup: degarelix 240/80 mg

Severe/life-threatening adverse event

Subgroup: abarelix $100 \mathrm{mg}$

Subgroup: degarelix 240/80 mg

Subgroup: degarelix 240/160 mg

Discontinuation due to adverse events

Subgroup: abarelix $100 \mathrm{mg}$

Subgroup: degarelix 240/80 mg

Subgroup: degarelix 240/160 mg

Fatigue

Subgroup: abarelix $100 \mathrm{mg}$

Subgroup: degarelix 240/80 mg and 240/160 mg

Hot flush

Subgroup: abarelix $100 \mathrm{mg}$

Subgroup: degarelix 240/80 mg and 240/160 mg

Infection (abarelix $100 \mathrm{mg}$ )

Urinary tract infection

Subgroup: abarelix $100 \mathrm{mg}$

Subgroup: degarelix 240/80 and 240/160 mg

Loss of sexual interest

Subgroup: abarelix $100 \mathrm{mg}$

Subgroup: degarelix 240/80 mg

Sexual dysfunction (degarelix 240/80 mg)

Acute myocardial infarction

Subgroup: degarelix 240/160 mg

Subgroup: degarelix 240/80 mg

Cardiovascular events (degarelix 240/80 and 240/160 mg)

Ischaemic heart disease

Subgroup: degarelix 240/160 mg

Subgroup: degarelix 240/80 mg

Fatal cerebrovascular-related events (degarelix 240/80 mg and 240/160 mg)

Asthenia (degarelix 240/80 mg)

Urinary retention

Subgroup: degarelix 240/160 mg

Subgroup: degarelix 240/80 mg

Immediate onset allergic reactions $(<1 \mathrm{~h})$ (abarelix $100 \mathrm{mg})$

Injection-site pain degarelix 240/80 mg and 240/160 mg

Injection-site reaction (degarelix 240/80 mg and 240/160 mg)

Diarrhoea (abarelix $100 \mathrm{mg}$ )

Peripheral oedema (abarelix $100 \mathrm{mg}$ )

Constipation

Subgroup: abarelix $100 \mathrm{mg}$

Subgroup: degarelix 240/160 mg

Subgroup: degarelix 240/80 mg

Arthralgia

Subgroup: abarelix $100 \mathrm{mg}$

Subgroup: degarelix 240/80 mg and 240/160 mg

Musculoskeletal adverse events (degarelix 240/80 mg)

Chills

Subgroup: degarelix 240/80 mg

Subgroup: degarelix 240/160 mg

Back pain

Subgroup: abarelix $100 \mathrm{mg}$

Subgroup: degarelix 240/80 mg and 240/160 mg

*Statistical method: Mantel-Haenszel, fixed-effect model.

†Statistical method: Mantel-Haenszel, random-effects model.

¥Statistical method: Generic inverse variance, fixed-effect model.

$\mathrm{MD}$, mean difference; NA, not applicable; RR, risk ratio.
Effect estimate(95\% Cl),

Studies Patients heterogeneity (12)

$7 \quad 2179$

$3 \quad 1102$

1302

$4 \quad 775$

$5 \quad 2064$

$4 \quad 1454$

308

302

2290

1110

872

308

3784

1456

2328

3264

936

2328

520

2848

520

2328

597

352

245

427

610

302

308

2328

610

302

308

610

427

1077

302

775

1694

2328

610

872

520

1522

872

303

347

2680

352

2328

408

610

308

302

3200

872

2328
RR 0.82 (0.62 to 1.08$), 4 \%{ }^{*}$ RR 0.88 (0.60 to 1.28$), 0 \%$ * RR 0.85 (0.46 to 1.57$), N^{*}$ RR 0.68 (0.39 to 1.19$), 35 \%$ * RR 0.76 (0.58 to 1.00$), 4 \%$ * RR 0.79 (0.60 to 1.05$), 0 \%$ * RR 0.16 (0.02 to 1.54$), \mathrm{NA}^{*}$ RR 0.50 (0.07 to 3.46$), \mathrm{NA}^{*}$ RR 0.86 (0.57 to 1.31$), 25 \%$ * RR 0.58 (0.31 to 1.08$), 39 \%$ * RR 0.95 (0.44 to 2.04$), 0 \%$ * RR 1.57 (0.65 to 3.81), NA* RR 0.88 (0.72 to 1.08$), 0 \%$ * RR 0.96 (0.73 to 1.26$), 0 \%$ * RR 0.80 (0.59 to 1.08$), N^{*}$ RR 1.00 (0.92 to 1.08$), 0 \%$ * RR 1.01 (0.93 to 1.10$), 0 \%$ * RR 0.99 (0.88 to 1.11), NA* RR 0.93 (0.42 to 2.05), NA* RR 0.71 (0.41 to 1.25$), 54 \% \dagger$ RR 1.03 (0.52 to 2.07), NA† RR 0.57 (0.39 to 0.83), NA† RR 1.05 (0.38 to 2.91$), 0 \%$ * RR 1.00 (0.06 to 15.86), NA* RR 1.06 (0.35 to 3.17$), \mathrm{NA}^{*}$ RR 0.83 (0.40 to 1.71$), 0 \%$ * RR 0.49 (0.07 to 3.48$), 0 \%$ * RR 1.49 (0.06 to 36.31), NA* RR 0.16 (0.01 to 3.98), NA* RR 0.60 (0.38 to 0.94$)$, NAł RR 0.42 (0.23 to 0.77$), 0 \%$ * RR 0.50 (0.21 to 1.15$), N^{*}$ * RR 0.35 (0.15 to 0.85$), N^{*}$ RR 0.49 (0.12 to 1.94$), N^{*}$ RR 0.91 (0.39 to 2.13 ), $0 \%$ * RR 0.39 (0.12 to 1.32$), 0 \%$ * RR 0.99 (0.09 to 10.79), NA* RR 0.28 (0.06 to 1.23 ), $0 \%$ * RR 2.36 (0.55 to 10.12$), 0 \%$ * RR 7.88 (5.65 to 10.98), NA* RR 79.61 (11.23 to 564.49), NA* RR 1.21 (0.81 to 1.80$), 0 \%$ * RR 0.51 (0.32 to 0.81$), \mathrm{NA}^{*}$ RR 0.99 (0.64 to 1.53$), 0 \%$ * RR 1.00 (0.58 to 1.75$), 0 \%$ * RR 0.60 (0.19 to 1.92$), N^{*}$ RR 1.28 (0.49 to 3.33$), 0 \%$ * RR 0.64 (0.45 to 0.91$), 0 \%$ * RR 0.40 (0.08 to 2.03), NA* RR 0.66 (0.46 to 0.94$), N^{*}$ RR 0.65 (0.45 to 0.96$), N^{*}$ RR 9.38 (1.26 to 69.58$), 0 \%$ * RR 11.28 (0.67 to 189.51), NA* RR 7.46 (0.43 to 129.37$), N^{*}$ RR 0.74 (0.56 to 0.97$), 4 \%$ * RR 0.81 (0.54 to 1.23 ), 38\%* RR 0.68 (0.48 to 0.99 ), NA* 
safety data analysis, treated with abarelix, discontinued therapy because of immediate onset of allergic-type adverse events, and $0.4-0.5 \%$ had serious anaphylacticlike reactions. There were no such events in the control groups treated with standard androgen suppression therapy. ${ }^{23}$ Additionally, the risk for injection-site events was increased using GnRH antagonists. This result is consistent with the FDA safety data analysis, where 25\% of patients treated with degarelix had injection-site reactions (grade 3 or 4 events in $1 \%$ of patients). ${ }^{51}$

Fewer cardiovascular events occurred among patients using GnRH antagonists than among patients using standard androgen suppression therapy. This has been noted in the literature previously. ${ }^{60}{ }^{77-79}$ However, there is evidence for both medications that in patients with a pre-existing cardiovascular disease and/or corresponding risk factors, these drugs may increase the risk to suffer from cardiovascular events in the long term and therefore, these subgroup of patients may need careful clinical follow-up. ${ }^{78-81}$

\section{CONCLUSION}

Evidence is hampered by risk of bias, selective reporting, and limited follow-up. Quality of evidence for all assessed outcomes was rated low according to GRADE. There is currently insufficient evidence to make firm conclusive statements on the efficacy of GnRH antagonist compared to standard androgen suppression therapy for advanced prostate cancer. The risk for injection-site events was increased, but cardiovascular events may occur less often using GnRH antagonist. Further highquality research on GnRH antagonists with long-term follow-up is required.

\section{Author affiliations \\ ${ }^{1}$ Department of Urology, University Hospital Erlangen, Erlangen, Germany \\ ${ }^{2}$ UroEvidence, Deutsche Gesellschaft für Urologie, Düsseldorf/Berlin, Germany \\ ${ }^{3}$ Department of Urology, University Hospital Frankfurt, Frankfurt, Germany \\ ${ }^{4}$ German Cochrane Centre, Medical Center-University of Freiburg, Freiburg, \\ Germany \\ ${ }^{5}$ Department of Obstetrics, Gynecology and Reproductive Medicine, Cairo University Hospital, Cairo, Egypt}

\section{Twitter Follow Joerg Meerpohl at @meerpohl}

Contributors FK, HB, AB, BK, BW, CS, DS, CR, SS, AW and JJM were involved in the conception or design of the work, or the acquisition, analysis or interpretation of data; and involved in the drafting the work or revising it critically for important intellectual content and gave final approval of the version. All of the authors have read and approved the manuscript. All of the authors had full access to all study data, and take full responsibility for the integrity of the data and the accuracy of the data analysis.

Funding This systematic review was supported by a grant from the German Federal Ministry of Education and Research (Bundesministerium für Bildung und Forschung, BMBF, 01KG1218).

Competing interests None declared.

Provenance and peer review Not commissioned; externally peer reviewed.

Data sharing statement Additional data can be accessed via the Dryad data repository at http://datadryad.org/ with the doi:10.5061/dryad.11k3m.
Open Access This is an Open Access article distributed in accordance with the Creative Commons Attribution Non Commercial (CC BY-NC 4.0) license, which permits others to distribute, remix, adapt, build upon this work noncommercially, and license their derivative works on different terms, provided the original work is properly cited and the use is non-commercial. See: http:// creativecommons.org/licenses/by-nc/4.0/

\section{REFERENCES}

1. Tomera K, Gleason D, Gittelman M, et al. The gonadotropin-releasing hormone antagonist abarelix depot versus luteinizing hormone releasing hormone agonists leuprolide or goserelin: initial results of endocrinological and biochemical efficacies in patients with prostate cancer. J Urol 2001;165:1585-9.

2. Trachtenberg J, Gittleman M, Steidle C, et al. A phase 3, multicenter, open label, randomized study of abarelix versus leuprolide plus daily antiandrogen in men with prostate cancer. J Urol 2002:1670-4.

3. Arai G, Nishio K, Sato R, et al. Possible clinical implication of serum testosterone surge caused by the $\mathrm{GnRH}$ antagonist degarelix. ASCO Meeting Abstr 2014;32(15_suppl):e16097.

4. Crawford ED, Tombal B, Miller K, et al. A phase III extension trial with a 1-arm crossover from leuprolide to degarelix: comparison of gonadotropin-releasing hormone agonist and antagonist effect on prostate cancer. J Urol 2011;186:889-97.

5. Shore ND, Abrahamsson PA, Anderson J, et al. New considerations for ADT in advanced prostate cancer and the emerging role of $\mathrm{GnRH}$ antagonists. Prostate Cancer Prostatic Dis 2013;16:7-15.

6. McLeod D, Zinner N, Tomera K, et al. A phase 3, multicenter open-label, randomized study of abarelix versus leuprolide acetate in men with prostate cancer. Urology 2001;58:756-61.

7. Hatoum HT, Crawford ED, Nielsen SK, et al. Cost-effectiveness analysis comparing degarelix with leuprolide in hormonal therapy for patients with locally advanced prostate cancer. Expert Rev Pharmacoecon Outcomes Res 2013;13:261-70.

8. Lee D, Porter J, Gladwell D, et al. A cost-utility analysis of degarelix in the treatment of advanced hormone-dependent prostate cancer in the United Kingdom. J Med Econ 2014;17:233-47.

9. Wolff J, Tolle A, Gedamke M. Health care cost in hormone-naive and hormonally pretreated patients with prostate cancer treated with degarelix. J Clin Oncol (Meeting Abstracts) 2012;30(Suppl 5):240.

10. Klotz L, Miller K, Crawford ED, et al. Disease control outcomes from analysis of pooled individual patient data from five comparative randomised clinical trials of degarelix versus luteinising hormone-releasing hormone agonists. Eur Urol 2014;66:1101-8.

11. Cui $\mathrm{Y}$, Zong $\mathrm{H}$, Yan $\mathrm{H}$, et al. Degarelix versus goserelin plus bicalutamide therapy for lower urinary tract symptom relief, prostate volume reduction and quality of life improvement in men with prostate cancer: a systematic review and meta-analysis. Urol Int 2014;93:152-9.

12. Koch M, Steidle C, Brosman S, et al. An open-label study of abarelix in men with symptomatic prostate cancer at risk of treatment with LHRH agonists. Urology 2003;62:877-82.

13. Heidenreich A, Bastian PJ, Bellmunt J, et al. Guidelines on prostate cancer. Uroweb 2013. (accessed 17 Feb 2014). http://www. uroweb.org

14. Higgins JPT, Altman DG, Sterne JAC. Chapter 8: Assessing risk of bias in included studies. In: Higgins JPT, Green S, eds. Cochrane handbook for systematic reviews of interventions Version 5.1.0 (updated March 2011). The Cochrane Collaboration, 2011. http:// www.cochrane-handbook.org

15. Reeves BC, Deeks JJ, Higgins JPT, et al. Chapter 13: Including non-randomized studies. In: Higgins JPT, Green S, eds. Cochrane handbook for systematic reviews of interventions. Chichester, UK: John Wiley \& Sons, 2008:391-432.

16. Guyatt G, Oxman AD, Akl EA, et al. GRADE guidelines: 1. Introduction-GRADE evidence profiles and summary of findings tables. J Clin Epidemiol 2011;64:383-94.

17. Schünemann HJ, Oxman AD, Vist GE, et al. Chapter 12: Interpreting results and drawing conclusions. In: Higgins JPT, Green S, eds. Cochrane handbook for systematic reviews of interventions Chichester, UK: John Wiley \& Sons, 2008:359-87.

18. Liberati A, Altman DG, Tetzlaff J, et al. The PRISMA statement for reporting systeamtic reviews and meta-analyses of studies that evaluate health care interventions: explanation and elaboration. PLoS Med 2009;6:e1000100.

19. Garnick MB, Tomera K, Campion M, et al. Abarelix-depot (A-D), a sustained release (SR) formulation of a potent $\mathrm{GnRH}$ pure antagonists in patients (pts) with prostate cancer (PrCA): phase II 
clinical results and endocrine comparison with agonists Lupron (L) and Zoladex (Z) (abstract). J Urol 1999;161(Suppl 4):340.

20. Garnick MB, Campion M. Abarelix Depot, a GnRH antagonist, v LHRH superagonists in prostate cancer: differential effects on follicle-stimulating hormone. Abarelix Depot study group. Mol Urol 2000;4:275-7.

21. McLeod D, Zinner N, Gleason D, et al. Abarelix-Depot (A-D) versus leuprolide acetate $(\mathrm{L})$ for prostate cancer: results of a multi-institutional, randomized, phase III study in 271 patients. Proc Am Soc Clin Oncol 2000;19:332a, Abstract 1306.

22. Garnick MB, Pratt CM, Campion M, et al. The effect of hormonal therapy for prostate cancer on the electrocardiographic QT interval: Phase 3 results following treatment with leuprolide and goserelin, alone or with bicalutamide, and the GnRH antagonist abarelix. J Clin Oncol 2004;22(14S (July 15 Suppl)):400, Abstract 4578

23. Center for Drug Evaluation and Research. Approval package for: Application number 21-320. 2003. http://www.accessdata.fda.gov/ drugsatfda docs/nda/2003/21-320 Plenaxis BioPharmr.pdf

24. Trachtenberg J, Gittelman M, Steidle C, et al. Abarelix-Depot (A-D) versus leuprolide acetate $(\mathrm{L})$ plus bicalutamide [Casodex $(\mathrm{C})$ ], for prostate cancer: results of a multi-institutional, randomized, phase III study in 255 patients. Proc Am Soc Clin Oncol 2000;19:332a, Abstract 1307

25. Trachtenberg J, Fotheringham N, Campion M. Avoidance of FSH surge and maintained suppression of follicle-stimulating hormone (FSH) with abarelix depot (A-D) compared to leuprolide (L) \pm bicalutamide in prostate cancer (PC) patients. Proc ASCO 2001;20:152 (Abstract 2358).

26. Fisher H, Barze II W, Gittelman M, et al. Abarelix depot (A-D) monotherapy reduces PSA levels comparable to leuprolide acetate (L) plus bicalutamide (B): results of a multicenter trial of rising PSA, advanced (D1/D2), neoadjuvant hormonal therapy (NHT), and intermittant hormonal therapy (IHT) prostate cancer (PC) patients (pts. Proc Am Soc Clin Oncol 2001;20:152b, Abstract 2359.

27. Gittelman M, Gleave M, Pommerville PJ, et al. Greater and more rapid decrease in prostate specific antigen (PSA) and testosterone (T) levels with abarelix depot (A-D) compared to leuprolide acetate (L): Results of a multicenter 24-week safety study. Proc ASCO 2001;20:154 (Abstract 2367)

28. Garnick M, Pratt C, Campion M, et al. Increase in the electrocardiographic QTC interval in men with prostate cancer undergoing androgen deprivation therapy: Results of three randomized controlled clinical studies. Eur Urol Supp/ 2004;3:57.

29. Debruyne F, Bhat G, Garnick MB. Abarelix for injectable suspension: first-in-class gonadotropin-releasing hormone antagonist for prostate cancer. Future Oncol 2006;2:677-96.

30. Debruyne FM. Gonadotropin-releasing hormone antagonist in the management of prostate cancer. Rev Urol 2004;6(Suppl 7):S25-32.

31. Garnick MB, Mottet N. New treatment paradigm for prostate cancer: abarelix initiation therapy for immediate testosterone suppression followed by a luteinizing hormone-releasing hormone agonist. BJU Int 2012;110:499-504.

32. Albertsen $\mathrm{P}$, Tombal B, Wiegel T, et al. Androgen deprivation therapy by a gonadotropin releasing hormone antagonist, degarelix, lowers the risk of cardiovascular events or death when compared to luteinising hormone-releasing agonists. J Urol 2013;189:e322.

33. Albertsen PC. Comparision of the risk of cardiovascular events and death in patients treated with degarelix compared with $\mathrm{LHRH}$ agonists. J Clin Oncol (Meeting Abstracts) 2013;31(Suppl 6):42.

34. Miller K, Tombal B, Albertsen $\mathrm{P}$, et al. Risiko für kardiovaskuläre Ereignisse und Tod geringer bei Patienten, die mit Degarelix im Vergleich zu LHRHAgonisten behandelt wurden. Urologe 2013;52 (Suppl 1):78 (V24.8)

35. Crawford ED, Shore N, Miller K, et al. Degarelix versus LHRH agonists: differential skeletal and urinary tract outcomes from an analysis of six comparative randomized clinical trials. J Clin Oncol (Meeting Abstracts) 2013;31(Suppl 6):68.

36. Klotz L, Boccon-Gibod L, Shore ND, et al. The efficacy and safety of degarelix: a 12-month, comparative, randomized, open-label, parallel-group phase III study in patients with prostate cancer. BJU Int 2008:102:1531-8.

37. Tombal B, Miller K, Boccon-Gibod L, et al. Additional analysis of the secondary end point of biochemical recurrence rate in a phase 3 trial (CS21) comparing degarelix $80 \mathrm{mg}$ versus leuprolide in prostate cancer patients segmented by baseline characteristics. Eur Urol 2010;57:836-42.

38. Schröder FH, Boccon-Gibod L, Tombal B, et al. Degarelix versus luprolide in patients with prostate cancer: effects in metastatic patients as assessed by serum alkaline phosphatase. Eur Uro Suppl 2009;8:130.
39. Gittelman M, Shore N, Jensen J, et al. Degarelix versus leuprolide treatment in patients with advanced prostate cancer (Pca): PSA failures during a randomized, phase III trial (CS21). Genitourin Cancer Symp Am Soc Clin Oncol 2009:Abstract 209, http:// meetinglibrary.asco.org/

40. Boccon-Gibod L, Klotz L, Schroder $\mathrm{H}$, et al. Degarelix compared to leuprolide depot $7.5 \mathrm{mg}$ in a 12-month randomised, open-label, parallel-group phase III study in prostate cancer patients. Eur Urol Suppl 2008;7:205.

41. Damber J-E, Tammela TLJ, Iversen $P$, et al. The effect of baseline testosterone on the efficacy of degarelix and leuprolide: further insights from a 12-month, comparative, phase III study in prostate cancer patients. Urology 2012;80:174-80.

42. Tombal B, Miller K, Boccon-Gibod L, et al. Degarelix versus leuprolide in prostate cancer patients: new prostate-specific antigen data from a phase III trial (CS21). EJC Supp/ 2009;7:411.

43. Tombal B, Miller K, Boccon-Gibod L, et al. Degarelix Vs. Leuprolide Treatment in Patients with Advanced Prostate Cancer: Psa Failures during a Randomised, Phase lii Trial (Cs21). Eur Urol Suppl 2009;8:130

44. Smith MR, Klotz L, Persson BE, et al. Cardiovascular safety of degarelix: results from a 12-month, comparative, randomized, open label, parallel group phase III trial in patients with prostate cancer. $J$ Urol 2010;184:2313-9.

45. Schroder FH, Tombal B, Miller K, et al. Changes in alkaline phosphatase levels in patients with prostate cancer receiving degarelix or leuprolide: results from a 12-month, comparative, phase III study. BJU Int 2010;106:182-7.

46. Schroeder F, Boccon-Gibod L, Tombal B, et al. Degarelix versus leuprolide in patients with metastatic prostate cancer: assessment of serum alkaline phosphatase over time. EJC Supp/ 2009;7:411.

47. Boccon-Gibod L, Klotz L, Schroder FH, et al. Efficacy and safety of degarelix versus leuprolide depot $(7.5 \mathrm{mg})$ in a 12-month, randomized, open-label, phase III study in patients with prostate cancer. Ann Oncol 2009;(Suppl 8):198; Abstract 614P.

48. Moul JW, Crawford E, Shore N, et al. PSA and serum alkaline phosphatase (S-ALP) control in patiens with prostate cancer (PCa) receiving degarelix or leuprolide. Genitourin Cancer Symp Am Soc Clin Oncol 2010:Abstract 111. http://meetinglibrary.asco.org/

49. Schröder FH, Tombal B, Boccon-Gibod L, et al. Degarelix vs leuprolide treatments in patients with advanced prostate cancer: PSA failures and effects in S-ALP levels during a randomised, phase III trial (CS21). Eur Multidisciplinary Meeting Urol Cancers 2009. http://www.uroweb.org/events/abstracts-online/

50. Damber JE, Tammela T, Abrahamsson PA, et al. Comparing testosterone and PSA for different baseline testosterone concentrations during initiation of degarelix and leuprolide treatment. Eur Urol Suppl 2009;8:130.

51. Center for Drug Evaluation and Research. Application Number: 22-201. 2008. http://www.accessdata.fda.gov/drugsatfda_docs/nda/ 2008/022201s000_MedR.pdf

52. Shore ND, Moul JW, Crawford E, et al. Prostate-specific antigen (PSA) progression-free survival (PFS): a comparison of degarelix versus leuprolide in patients with prostate cancer. $J$ Clin Oncol (Meeting Abstracts) 2011;29(Suppl 7):12.

53. Klotz L, Smith M, Persson B, et al. Cardiovascular safety of degarelix: Results from a 12-month, comparative, randomized, open-label, parallel-group phase III trial in prostate cancer patients. J Urol 2010;183(4 Suppl):e228; Abstract 582.

54. Boccon-Gibod L, Klotz L, Schröder FH, et al. Efficacy and Safety of Degarelix Versus Leuprolide Depot $(7.5 \mathrm{mg})$ in a 12-Month, Randomized, Open-Label, Phase III Study in Patients with Prostate Cancer. Ann Oncol 2008;19(Suppl 8):198.

55. Crawford ED, Moul JW, Shore ND, et al. Prostate-specific antigen and serum alkaline phosphatase levels in prostate cancer patients receiving Degarelix or leuprolide. J Urol 2010;183:e338.

56. Iversen P, Damber JE, Malmberg A, et al. Improved outcomes with degarelix monotherapy compared with luteinizing hormone-releasing hormone (LHRH) agonists plus antiandrogen flare protection in the treatment of men with advanced prostate cancer. Scand J Urol 2013;47:7

57. Iversen $\mathrm{P}$, Karup C, van der Meulen E, et al. Hot flushes in prostatic cancer patients during androgen-deprivation therapy with monthly dose of degarelix or leuprolide. Prostate Cancer Prostatic Dis 2011:14:184-90.

58. Iversen $\mathrm{P}$, Karup C, van der Meulen EA, et al. Hot flushes (HF) during androgen deprivation therapy: direct comparison of monthly degarelix and leuprolide in a phase 3 trial. Congress Eur Soc Med Oncol 2010:Abstract: 3471. http://www.esmo.org/

59. Tombal B, Damber J-E, Malmberg A, et al. Degarelix monotherapy versus luteinizing hormone-releasing hormone (LHRH) agonists plus 
antiandrogen flare protection in the treatment of men with advanced prostate cancer. J Clin Oncol (Meeting Abstracts) 2014; 32(Suppl 4):86.

60. Tombal B, Albertsen P, De La Taille A, et al. Lower risk of cardiovascular (CV) events and death in men receiving ADT by gonadotropin releasing hormone $(\mathrm{GnRH})$ antagonist, degarelix, compared with luteinising hormone-releasing (LHRH) agonists. Annu Congress Eur Assoc Urol 2013. http://www.uroweb.org/events/ abstracts-online/

61. Miller K, Crawford ED, Shore N, et al. Disease control-related outcomes from an analysis of six comparative randomised clinical trials of degarelix versus luteinising hormone-releasing hormone (LHRH) agonists. Annu Congress Eur Assoc Urol 2013. http://www. uroweb.org/events/abstracts-online/

62. Shore N, Miller K, Tombal B, et al. Analysis of disease control-related outcomes from six comparative randomised clinical trials of degarelix versus luteinising hormone-releasing hormone agonists. J Urol 2013;189:e294.

63. Lee D, Nielsen SK, van Keep M, et al. Quality of life improvement in patients treated with degarelix versus leuprorelin for advanced prostate cancer. J Urol 2015;193:839-46.

64. Anderson J, Al-Ali G, Wirth M, et al. Degarelix versus goserelin (plus antiandrogen flare protection) in the relief of lower urinary tract symptoms secondary to prostate cancer: results from a phase IIllb study (NCT00831233). Urol Int 2013;90:321-8.

65. Mason MD, Bosnyak Z, Malmberg A, et al. Lower urinary tract symptoms (LUTS) in prostate cancer (PC) patients treated with GnRH antagonist compared to agonist: results of a pooled analysis. ASCO Meeting Abstr 2014;32(15_suppl):e16017.

66. Manka L, Wiegel T, Mason M, et al. Stronger short-term relief of symptoms of lower urinary tract (LUTS) in patients with prostate cancer of all stages after treatment with degarelix compared to goserelin/bicalutamide: results of a summarized Analysis. Strahlenther Onkol 2014:190:136-6.

67. Mason M, Maldonado Pijoan X, Steidle C, et al. Neoadjuvant androgen deprivation therapy for prostate volume reduction, lower urinary tract symptom relief and quality of life improvement in men with intermediate-to high-risk prostate cancer: a randomised non-inferiority trial of degarelix versus goserelin plus bicalutamide. Clin Oncol 2013;25:190-6.

68. Mason M, Steidle CP, Deliveliotis C, et al. Degarelix as neoadjuvant hormone therapy in patients with prostate cancer: Results from a phase IIIb randomized, comparative trial versus goserelin plus bicalutamide. J Clin Oncol 2012;30(Suppl 15):e15199.
69. Axcrona K, Aaltomaa S, Da Silva CM, et al. ADT for volume reduction, symptom relief and quality of life improvement in men with prostate cancer: degarelix versus goserelin plus bicalutamide. Eur Urol Suppl 2012;11:e985, e85a.

70. Axcrona K, Aaltomaa S, da Silva CM, et al. Androgen deprivation therapy for volume reduction, lower urinary tract symptom relief and quality of life improvement in patients with prostate cancer: degarelix vs goserelin plus bicalutamide. BJU Int 2012;110:1721-8.

71. Zuckerman JM, Eure G, Malcolm J, et al. Prospective evaluation of testosterone fluctuations during a transition of therapy from degarelix to leuprolide in patients on androgen deprivation therapy. Urology 2014;83:670-4.

72. Zuckerman J, Given R. Degarelix induction followed by leuprolide maintenance: a new treatment paradigm? J Urol 2013;189:e322-e23.

73. de la Rosette J, Davis R III, Frankel D, et al. Efficacy and safety of androgen deprivation therapy after switching from monthly leuprolide to monthly degarelix in patients with prostate cancer. Int $J$ Clin Pract 2011;65:559-66.

74. Moul JW. Prostate cancer: making the switch from LHRH antagonist to LHRH agonist. Nat Rev Urol 2012;9:125-6.

75. Tombal B, Schroder F, Miller K, et al. Long-term prostate specific antigen (Psa) control in prostate cancer: continuous degarelix or degarelix following leuprolide. Eur Urol Suppl 2011;10:335-5.

76. Crawford ED, Shore ND, Moul JW, et al. Long-term tolerability and efficacy of degarelix: 5-year results from a phase III extension trial with a 1-arm crossover from leuprolide to degarelix. Urology 2014;83:1122-8.

77. Albertsen PC, Klotz L, Tombal B, et al. Cardiovascular morbidity associated with gonadotropin releasing hormone agonists and an antagonist. Eur Urol 2014;65:565-73.

78. Smith MR, Klotz L, van der Meulen E, et al. Gonadotropin-releasing hormone blockers and cardiovascular disease risk: analysis of prospective clinical trials of degarelix. $J$ Urol 2011;186:1835-42.

79. Smith MR, Klotz L, van der Meulen E, et al. Association of baseline risk factors with cardiovascular (CV) events during long-term degarelix therapy in men with prostate cancer. J Clin Oncol 2011;29 (Suppl 7):3705-14. Abstract 190

80. Efstathiou JA, Bae K, Shipley WU, et al. Cardiovascular mortality after androgen deprivation therapy for locally advanced prostate cancer: RTOG 85-31. J Clin Oncol 2009;27:92-9.

81. Efstathiou JA, Bae K, Shipley WU, et al. Cardiovascular mortality and duration of androgen deprivation for locally advanced prostate cancer: analysis of RTOG 92-02. Eur Urol 2008;54:816-23. 